\title{
An algorithm for adaptive introduction and arrangement of velocity discontinuities within 3D finite-element-based upper bound limit analysis approaches
}

\author{
M. Li ${ }^{\mathrm{a}, *}$, J. Füssla ${ }^{\mathrm{a}}$, M. Lukacevic ${ }^{\mathrm{a}}$, J. Eberhardsteiner ${ }^{\mathrm{a}}$, C.M. Martin ${ }^{\mathrm{b}}$ \\ ${ }^{a}$ Vienna University of Technology, Karlsplatz 13/202, Vienna 1040, Austria \\ ${ }^{b}$ University of Oxford, Parks Road, Oxford OX1 3PJ, UK
}

\begin{abstract}
This paper presents a new adaptive strategy to efficiently exploit velocity discontinuities in 3D finite-element-based upper bound limit analysis formulations. Based on an initial upper bound result, obtained with a conventional approach without velocity discontinuities, possible planes of plastic flow localisation are determined at each strain-rate evaluation node and, subsequently, this information is used to sequentially introduce discontinuities into the considered discretised structure. During a few iterations, by means of introducing new and adjusting existing discontinuities, an optimal velocity discontinuity layout is obtained. For the general 3D case, the geometry of this layout is defined by the well-known level-set method, standardly used to define the geometry of cracks in extended finite element method.

To make this method also applicable for orthotropic strength behaviours, traction-based yield functions defining the plastic flow across discontinuities are derived form their stress-based counterparts. This procedure is outlined in detail and the obtained traction-based yield functions are verified numerically, to guarantee a consistent strength behaviour throughout the whole discretised structure.

By means of three different examples, including isotropic as well as orthotropic yield functions, the performance of the proposed strategy is investigated and upper bound results as well as failure modes are compared to reference solutions. The proposed approach delivered reliable upper bounds for each example and the majority of plastic flow takes place across the sensibly-arranged discontinuities. For this reason, very good upper bounds can be obtained with a quite coarse finite element mesh and only few introduced velocity discontinuities. This represents an attractive alternative to commonly-used adaptive mesh refinement strategies, where often a huge amount of degrees of freedom needs to be added to capture localised failure.
\end{abstract}

Keywords: adaptive strategy, velocity discontinuities, upper bound limit analysis, level-set method, orthotropic strength behaviours, localised failure

\section{Introduction}

Limit analysis has been proven as a powerful computational method for strength predictions of a wide range of materials used in practical engineering areas, such as soil, masonry, and composite materials $[1,2,3,4,5,6]$. Originally, the main objective of limit analysis has been determining the ultimate load a structure, exhibiting elastoplastic material response, can resist without structural collapse. Since structures lose the capability to store further external work as recoverable internal energy at structural collapse, limit analysis exclusively focuses on the time instant when the critical plastic dissipation rate is achieved.

This problem can be stated as: Find the kinematically admissible velocity field which minimises the energy dissipation rate over the set of all statically admissible stress fields which maximise the dissipated energy, according to Ciria et al. [7]. Based on the assumptions of small deformations, perfectly plastic material behaviour, and associated plastic

\footnotetext{
${ }^{*}$ Corresponding author.

Email address: mingjing. li@tuwien.ac.at (M. Li)
} 
flow, the plastic bounding theorems by Drucker el at. [8,9] are normally employed, leading to a lower and an upper bound of the collapse load multiplier. The lower bound theorem states that, the highest load supported by a statically admissible stress field, which satisfies equilibrium, the static boundary condition, and a plastic yield criterion, is an optimal lower bound on the exact collapse load. In contrast, the upper bound theorem states that, when the velocity field is kinematically admissible by satisfying compatibility, the kinematic boundary conditions and an associated plastic flow rule, the lowest load determined by equating the external work rate to the internal plastic dissipation rate is an optimal upper bound on the exact collapse load. Note that this paper mainly focuses on the upper bound problem.

Since analytical solutions of limit analysis are normally limited to simple problems, the limit analysis theorems have been successfully implemented in numerical formulations since the early 70s and excellent practical capabilities have been achieved in such numerical approaches, from simple two-dimensional (2D) benchmark examples to complicated three-dimensional (3D) problems. Time efficiency and solution quality of these approaches have also benefited from the rapid development of mathematical programming methods used to solve the underlying optimisation problems.

In early implementations, during the last three decades of the 20th Century, these optimisation problems were solved using linear programming methods by linearising the applied plastic yield functions, as can be found in [10, $11,12,13,14,15,16]$. Lyamin and Sloan [17, 18] and Krabbenhøft and Damkilde [19] were the pioneers who used nonlinear programming methods to solve numerical limit analysis formulations with nonlinear yield functions formulated in their native form. However, since such implementations require differentiability of the yield surfaces, local smoothening procedures are often necessary. Alternatively, as proposed by Makrodimopoulos and Martin [20, 21], Ciria and Peraire [22], and Krabbenhøft et al. [23], second-order cone programming (SOCP) has been proven to be a robust and efficient method to solve large-scale nonlinear optimisation problems of numerical limit analysis, allowing most commonly-used yield functions to be formulated in their native form. Also in this work SOCP is employed, since all used yield functions can be formulated as second-order cones, including the isotropic von Mises yield function, the Drucker-Prager yield function, as well as the orthotropic Tsai-Wu yield function.

On the other hand, the performance of numerical limit analysis formulations also heavily depends on the discretisation method and the related shape functions. Since rigorous upper bound solutions require the associated plastic flow rule being satisfied at each point of the structures, it is straightforward to use constant strain elements $[2,15,18,22,24]$. To achieve a higher quality of upper bound solutions, the simplex strain element using linear shape functions for the strain-rate field has been introduced by Makrodimopoulos and Martin [21, 25]. In their implementations, associated plastic flow can still be guaranteed within each element since the strain-rate is linear and the associated plastic flow rule is evaluated at all vertices. From the numerical point of view, further performance improvements can be expected by using higher order interpolation functions. For this purpose, several different implementations were developed using the element-free Galerkin method by Le et al. [26, 27], the radial point interpolation meshless method by Liu and Zhao [28] and Yu et al. [29], and the smoothed finite element method by Le et al. [30] and Nguyen-Xuan et al. [31]. However, in such implementations, it is difficult to guarantee both compatibility and satisfaction of the associated plastic flow rule in each point of the structure, thus, the so-obtained numerical upper bounds are not necessarily rigorous.

Beside using higher order interpolation functions, velocity discontinuities can be implemented into numerical limit analysis formulations, adding additional degrees of freedom to the discretised structure. In an early implementation by Sloan and Kleeman [16], velocity discontinuities were introduced in finite-element-based upper bound formulations where linear velocity jumps were allowed between constant strain elements introduced in [15]. As a development of this approach, zero-thickness interface elements were introduced by Krabbenhøft et al.[32], allowing the use of general yield criteria. In a contribution by Makrodimopoulos and Martin [33], velocity discontinuities were introduced between simplex strain elements, defined in [21], allowing both linear and quadratic velocity jumps, showing a robust and efficient performance for a large number of applications.

Alternative approaches of numerical upper bound formulations were proposed by Chen et al. [34] and Milani et al.[3, 4, 35], where all solid elements are considered as rigid and plastic failure is only possible across velocity discontinuities between elements. Such implementations exhibit good performance for certain engineering problems, e.g. in masonry engineering, but the strong dependence of the solution quality on the finite element mesh restricts its application, and even adaptive mesh refinement cannot fully compensate this drawback. For this reason, a sequential linear programming method was applied by Milani and Lourenço [36], to iteratively adapt the finite element mesh. This approach is able to increase the efficiency of velocity discontinuities, but so far it has been proposed only for $2 \mathrm{D}$ 
problems and the original mesh has to be fine enough to provide a sufficient number of velocity discontinuities, since no additional velocity discontinuity can be introduced during the adaptive iterations. Another interesting approach is the so-called discontinuity layout optimisation, presented by Smith and Gilbert [37] for 2D and Hawksbee et al. [38] for 3D problems. Thereby, velocity discontinuities are determined by using a truss layout optimisation algorithm based on a prescribed grid. Although this approach performs well for $2 \mathrm{D}$ problems, the determination of complex failure mechanisms in 3D solid bodies requires a very fine grid and large computational effort.

For this reason, in the author's opinion, the most promising approach so far to obtain rigorous upper bounds seems to be the use of solid finite elements with or without velocity discontinuities. Due to the fact that plastic failure often occurs very localised, adaptive mesh refinement has been intensively investigated in [7, 22, 31, 39, 40, 41, 42, 43]. However, such strategies normally lead to very fine meshes in failure regions and, thus, increase the number of degrees of freedom and computational effort significantly.

Recently, an alternative approach has been presented by the authors [44], where finite-element-based upper bound formulations with sensibly-arranged velocity discontinuities have been proven reliable and efficient, without the need of intensive mesh refinement in failure regions. Additionally, although the implementation of orthotropic yield functions in numerical upper bound formulations has been presented in several publications $[45,46,47,48,49,50]$, to the authors' knowledge, the combination of orthotropic yield functions and velocity discontinuities was presented by the authors in [44] for the first time. In order to apply this approach to more general problems, in this paper, a comprehensive introduction on the numerical implementations as well as an adaptive introduction and adjustment strategy on the sensible arrangement of velocity discontinuities will be presented.

Thus, the main objectives of this work can be introduced as follows:

1. The implementation of orthotropic yield functions and velocity discontinuities in 3D finite-element-based upper bound formulations, using simplex strain elements with the plastic flow measured by both the linear strain-rates within solid elements and linear velocity-jumps only across sensibly-arranged velocity discontinuities.

2. The derivation of traction-based yield functions for the velocity discontinuities, taking both their orientation and the orthotropic stress-based yield function assigned to the solid elements into account, in order to guarantee consistent orthotropic strength behaviour throughout the whole discretised structure.

3. The implementation of an adaptive approach to introduce and adjust velocity discontinuities in an efficient and meaningful way at regions where very localised plastic failure can be expected.

With respect to these objectives, the paper is structured as follows: In Section 2, upper bound formulations considering orthotropic strength behaviour and velocity discontinuities are introduced analytically. In these formulations, the traction-based yield function applied to velocity discontinuities are guaranteed to represent similar orthotropic strength behaviour as the stress-based yield function applied to solid elements by means of a projection algorithm introduced in Section 3. The formulation of associated plastic flow rule both in continuum and across velocity discontinuities are presented in Section 4. Then, in Section 5, upper bound formulations are given in discretised form, using simplex strain elements and linear velocity discontinuities. The algorithm for introduction and adjustment of velocity discontinuities is presented in Section 6. A verification of these implementations by means of two commonly-used benchmark examples under plane strain conditions and a 3D example can be found in Section 7. Finally, a brief summary and concluding comments are given in Section 8.

\section{Kinematic theorem}

Upper bound calculations exclusively focus on the kinematically admissible velocity field $\dot{\mathbf{u}}=\left(\dot{u}_{x}, \dot{u}_{y}, \dot{u}_{z}\right)^{\top} \in \mathfrak{R}^{3}$ at the time instant of structural collapse, and search for the failure mechanism leading to the minimum internal plastic energy dissipation rate $W_{\text {int }}$, which must equal to the external work rate $W_{\text {ext }}$. Thereby, a velocity field $\dot{\mathbf{u}}$ is considered to be kinematically admissible when it fulfills compatibility, the plastic flow rule, and the kinematic boundary conditions at each point of the considered structure.

In this work, to be able to consider very localised plastic failure mechanisms more easily, the velocity field $\dot{\mathbf{u}}$ is not enforced to be continuous, rather velocity-jumps $\Delta \dot{\mathbf{u}}=\left(\Delta \dot{u}_{x}, \Delta \dot{u}_{y}, \Delta \dot{u}_{z}\right)^{\top} \in \mathfrak{R}^{3}$ are allowed across prescribed discontinuities $\Gamma_{d i s}$. These consist of one part denoted as $\Gamma_{d i s}^{+}$with the related velocity field $\dot{\mathbf{u}}_{d i s}^{+}$and an opposite part $\Gamma_{d i s}^{-}$with the related velocity field $\dot{\mathbf{u}}_{d i s}^{-}$. Then, diffused plastic failure is measured by the plastic strain-rate field 
$\dot{\varepsilon}=\left(\dot{\varepsilon}_{x x}, \dot{\varepsilon}_{y y}, \dot{\varepsilon}_{z z}, 2 \dot{\varepsilon}_{x y}, 2 \dot{\varepsilon}_{y z}, 2 \dot{\varepsilon}_{x z}\right)^{\top} \in \Re^{6}$ (in Voigt notation) in the continuum $\Omega$ and very localised plastic failure is measured by the velocity-jump field $\Delta \dot{\mathbf{u}}=\dot{\mathbf{u}}_{d i s}^{+}-\dot{\mathbf{u}}_{d i s}^{-}$at certain discontinuities $\Gamma_{d i s}$.

The plastic dissipation functions for these two failure mechanisms can be expressed as

$$
\begin{array}{rlr}
d_{\text {mat }}(\dot{\boldsymbol{\varepsilon}})=\sup _{\boldsymbol{\sigma} \in \mathcal{F}} \boldsymbol{\sigma}^{\boldsymbol{\top}} \dot{\boldsymbol{\varepsilon}}, & \mathcal{F}=\left\{\boldsymbol{\sigma} \mid f_{\text {mat }}(\boldsymbol{\sigma}) \leq 0\right\} & \text { in } \Omega \\
d_{\text {dis }}(\Delta \dot{\mathbf{u}})=\sup _{\mathbf{t} \in \mathcal{D}} \mathbf{t}^{\top} \Delta \dot{\mathbf{u}}, & \mathcal{D}=\left\{\mathbf{t} \mid f_{\text {dis }}(\mathbf{t}) \leq 0\right\} & \text { on } \Gamma_{\text {dis }}
\end{array}
$$

where $\boldsymbol{\sigma}=\left(\sigma_{x x}, \sigma_{y y}, \sigma_{z z}, \sigma_{x y}, \sigma_{y z}, \sigma_{x z}\right)^{\top} \in \mathfrak{R}^{6}$ denotes the stress field, $\mathbf{t}=\left(t_{x}, t_{y}, t_{z}\right)^{\top} \in \mathfrak{R}^{3}$ represents the surface traction field, $f_{\text {mat }}(\boldsymbol{\sigma}) \leq 0$ is a stress-based yield function assigned to the continuum $\Omega$, and $f_{\text {dis }}(\mathbf{t}) \leq 0$ is a tractionbased yield function (depending on $f_{\text {mat }}(\sigma) \leq 0$ ) assigned to the velocity discontinuities $\Gamma_{\text {dis }}$. Consequently, the internal energy dissipation rate $W_{\text {int }}$ over the whole structure reads

$$
W_{i n t}=\int_{\Omega} d_{m a t}(\dot{\boldsymbol{\varepsilon}}) \mathrm{d} V+\int_{\Gamma_{\text {dis }}} d_{d i s}(\Delta \dot{\mathbf{u}}) \mathrm{d} A=\int_{\Omega} \dot{\boldsymbol{\varepsilon}}^{\top} \sigma \mathrm{d} V+\int_{\Gamma_{d i s}} \Delta \dot{\mathbf{u}}^{\top} \mathbf{t} \mathrm{d} A
$$

consisting of the rate of plastic energy dissipated within the continuum as well as at discontinuities.

The upper bound problem can then be formulated as a nonlinear optimisation problem, reading

$$
\begin{array}{lll}
\min & W_{\text {int }} & \\
\text { s.t. } & \dot{\boldsymbol{\varepsilon}}=\mathbf{L}_{\dot{\varepsilon}} \dot{\mathbf{u}} & \text { in } \Omega \\
& \Delta \dot{\mathbf{u}}=\mathbf{L}_{\Delta \dot{u}} \dot{\mathbf{u}}_{d i s} & \text { on } \Gamma_{d i s} \\
& \dot{\mathbf{u}}=\dot{\mathbf{u}}_{b c} & \text { on } \Gamma \\
& \dot{\boldsymbol{\varepsilon}}=\dot{\lambda}_{\text {mat }} \partial f_{\text {mat }}(\boldsymbol{\sigma}) / \partial \boldsymbol{\sigma} & \text { in } \Omega \\
& \Delta \dot{\mathbf{u}}=\dot{\lambda}_{d i s} \partial f_{d i s}(\mathbf{t}) / \partial \mathbf{t} & \text { on } \Gamma_{d i s}
\end{array}
$$

where $\dot{\mathbf{u}}_{d i s}=\left(\dot{\mathbf{u}}_{d i s}^{+}, \dot{\mathbf{u}}_{d i s}^{-}\right)^{\top} \in \Re^{6}$ contains the velocity fields of both surfaces of a discontinuity $\Gamma_{d i s}$, and

$$
\mathbf{L}_{\dot{\varepsilon}}=\left(\begin{array}{ccc}
\partial / \partial x & 0 & 0 \\
0 & \partial / \partial y & 0 \\
0 & 0 & \partial / \partial z \\
\partial / \partial y & 0 & \partial / \partial z \\
\partial / \partial x & \partial / \partial z & 0 \\
0 & \partial / \partial y & \partial / \partial x
\end{array}\right) \in \Re^{6 \times 3} \quad \text { and } \quad \mathbf{L}_{\Delta \dot{u}}=\left(\begin{array}{ll}
\mathbf{I} & -\mathbf{I}
\end{array}\right) \in \Re^{3 \times 6}
$$

are the compatibility operators for the continuum $\Omega$ and the discontinuities $\Gamma_{d i s}$, respectively.

Through the first constraint of the upper bound formulation in Eq. (3) compatibility between the velocity field $\dot{\mathbf{u}}$ and the strain-rate field $\dot{\boldsymbol{\varepsilon}}$ is enforced at each point of the continuum $\Omega$; the second constraint enforces compatibility between the velocity-jump field $\Delta \dot{\mathbf{u}}$ and the velocity field $\dot{\mathbf{u}}$ across each discontinuity $\Gamma_{d i s}$; the third constraint represents the kinematic boundary conditions, prescribing a velocity field $\dot{\mathbf{u}}_{b c}$ on the surface $\Gamma=\partial \Omega$. The last two constraints ensure associated plastic flow in $\Omega$ as well as across $\Gamma_{d i s}$, where $\dot{\lambda}_{m a t}$ and $\dot{\lambda}_{d i s}$ denote the corresponding plastic multiplier rates, determining the magnitude of plastic flow.

According to duality in nonlinear programming, the primal form of the upper bound problem in Eq. (3) can also be formulated in dual form, reading

$$
\begin{array}{ll}
\max & W_{\text {ext }} \\
\text { s.t. } & \int_{\Omega} \dot{\boldsymbol{\varepsilon}}^{\top} \boldsymbol{\sigma} \mathrm{d} V+\int_{\Gamma_{d i s}} \dot{\boldsymbol{\mu}}^{\top} \mathbf{t} \mathrm{d} A=\int_{\Omega} \dot{\mathbf{u}}^{\top} \beta \mathbf{g}_{u} \mathrm{~d} V+\int_{\Omega} \dot{\mathbf{u}}^{\top} \mathbf{g}_{f} \mathrm{~d} V+\int_{\Gamma_{u}} \dot{\mathbf{u}}^{\top} \beta \mathbf{t}_{u} \mathrm{~d} A+\int_{\Gamma_{f}} \dot{\mathbf{u}}^{\top} \mathbf{t}_{f} \mathrm{~d} A \\
& f_{\text {mat }}(\boldsymbol{\sigma}) \leq 0 \quad \text { in } \Omega \\
& f(\mathbf{t})_{d i s} \leq 0 \quad \text { on } \Gamma_{\text {dis }}
\end{array}
$$


where the first constraint represents a weak form of equilibrium for the whole structure, with $\beta$ as an unknown collapse limit load multiplier. The objective function becomes the maximum external work rate in the dual formulation, which can be written as

$$
W_{e x t}=\int_{\Omega} \dot{\mathbf{u}}^{\top} \beta \mathbf{g}_{u} \mathrm{~d} V+\int_{\Gamma_{u}} \dot{\mathbf{u}}^{\top} \beta \mathbf{t}_{u} \mathrm{~d} A+\int_{\Omega} \dot{\mathbf{u}}^{\top} \mathbf{g}_{f} \mathrm{~d} V+\int_{\Gamma_{f}} \dot{\mathbf{u}}^{\top} \mathbf{t}_{f} \mathrm{~d} A
$$

containing contributions from the unknown body force field $\mathbf{g}_{u}=\left(g_{u, x}, g_{u, y}, g_{u, z}\right)^{\top} \in \mathfrak{R}^{3}$ and the fixed body force field $\mathbf{g}_{f}=\left(g_{f, x}, g_{f, y}, g_{f, z}\right)^{\top} \in \mathfrak{R}^{3}$ in the continuum $\Omega$, as well as from the unknown traction field $\mathbf{t}_{u}$ on the boundary surface part $\Gamma_{u}$ and the fixed traction field $\mathbf{t}_{f}$ on the boundary surface part $\Gamma_{f}$, with $\Gamma=\Gamma_{u} \cap \Gamma_{f}$.

\section{Traction-based plastic yield function}

As discussed in several publications [16, 32, 33], the implementation of velocity discontinuities in finite-elementbased upper bound formulations can significantly improve the quality of upper bound results and locking phenomena can often be avoided. The number of unknown variables, however, is greatly increased and, thus, a higher computational effort is required.

In existing finite-element-based upper bound formulations with velocity discontinuities exclusively shear-failurebased isotropic yield functions $f_{\text {mat }}(\sigma) \leq 0$ (e.g. the von Mises yield function, the Mohr-Coulomb yield function and the Drucker-Prager yield function) are assigned to the continuum $\Omega$. In this case, the formulation of an equivalent strength behaviour at discontinuities (between finite elements) is straightforward and leads to a function $f_{\text {dis }}\left(\sigma_{m}, \tau\right) \leq 0$ depending on the normal stress $\sigma_{m}$ and the shear stress $\tau$ at the discontinuity. Thus, this failure criterion can easily be linked to and expressed in terms of the traction forces acting at the discontinuity, giving a function $f_{\text {dis }}(\mathbf{t}) \leq 0$. However, this is only possible for shear-failure-based strength criteria and becomes much more complicated for other types of failure criteria, like e.g. orthotropic strength behaviour.

For this reason, in this section an algorithm is proposed which allows the projection of a quite general stressbased yield function $f_{\text {mat }}(\boldsymbol{\sigma}) \leq 0$ onto an equivalent traction-based yield function $f_{d i s}(\mathbf{t}) \leq 0$ with respect to a certain velocity discontinuity $\Gamma_{d i s}$. In this way a consistent strength behaviour throughout a structure with discontinuities can be achieved.

\subsection{Projection algorithm}

As stress-based yield function for the continuum $\Omega$ the following differentiable quadratic form will be considered, formulated with respect to the local coordinate basis of the material $\left(x^{\prime}-y^{\prime}-z^{\prime}\right)_{\text {mat }}$ :

$$
f_{m a t}\left(\boldsymbol{\sigma}_{l o c}^{m a t}\right)=\boldsymbol{\sigma}_{l o c}^{m a t} \mathbf{P}_{\sigma}^{m a t} \boldsymbol{\sigma}_{l o c}^{m a t}+\left(\frac{1}{2} \mathbf{F}_{\sigma}^{m a t,+\boldsymbol{\top}} \boldsymbol{\sigma}_{l o c}^{m a t}\right)^{2}-\left(1-\frac{1}{2} \mathbf{F}_{\sigma}^{m a t,-\boldsymbol{T}} \boldsymbol{\sigma}_{l o c}^{m a t}\right)^{2} \leq 0, \quad \text { in } \Omega
$$

where $\boldsymbol{\sigma}_{l o c}^{m a t}=\mathbf{R}_{\sigma}^{\text {mat }} \boldsymbol{\sigma}$ represents a stress state with respect to the local coordinate basis of the material, and the matrices $\mathbf{P}_{\sigma}^{m a t} \in \Re^{6 \times 6}, \mathbf{F}_{\sigma}^{m a t,+} \in \Re^{6}$ and $\mathbf{F}_{\sigma}^{m a t,-} \in \mathfrak{R}^{6}$ determine the type of yield function as well as strength parameters. Of course, when the special case of an isotropic yield function is considered, the introduction of a local coordinate basis for the material is not necessary and $\sigma_{l o c}^{m a t}=\sigma$.

The transformation matrix for stress vectors from the global coordinate basis $(x-y-z)$ to the material local coordinate basis $\left(x^{\prime}-y^{\prime}-z^{\prime}\right)_{\text {mat }}$ reads

$$
\begin{aligned}
& \mathbf{R}_{\sigma}^{m a t}= \\
& \left(\begin{array}{c}
n_{x^{\prime} x}^{\operatorname{mat} 2} \\
n_{y^{\prime} x}^{\text {mat } 2} \\
n_{z^{\prime} x}^{\text {mat } 2} \\
n_{x^{\prime} x}^{\text {mat }} n_{y^{\prime} x}^{\text {mat }} \\
n_{y^{\prime} x}^{\text {mat }} n_{z^{\prime} x}^{\text {mat }} \\
n_{x^{\prime} x}^{\text {mat }} n_{z^{\prime} x}^{\text {mat }}
\end{array}\right. \\
& n_{x^{\prime} y}^{m a t 2} \quad n_{x^{\prime} z}^{m a} \\
& n_{y^{\prime} y}^{\text {mat2 }} \quad n_{y^{\prime} z}^{\text {mat2 }} \\
& n_{z^{\prime} y}^{\text {mat } 2} \quad n_{z^{\prime} z}^{\text {mat2 }} \\
& n_{x^{\prime} z}^{m a t 2} \quad 2 n_{x^{\prime} x}^{m a t} n_{x^{\prime} y}^{m a t} \\
& 2 n_{y^{\prime} x}^{m a t} n_{y^{\prime} y}^{m a t} \\
& 2 n_{z^{\prime} x}^{m a t} n_{z^{\prime} y}^{m a t} \\
& n_{x^{\prime} y}^{m a t} n_{y^{\prime} y}^{m a t} \quad n_{x^{\prime} z}^{m a t} n_{y^{\prime} z}^{m a t} \\
& n_{y^{\prime} y}^{\text {mat }} n_{z^{\prime} y}^{\text {mat }} \quad n_{y^{\prime} z}^{\text {mat }} n_{z^{\prime} z}^{\text {mat }} \quad n_{y^{\prime} x}^{\text {mat }} n_{z^{\prime} y}^{\text {mat }}+n_{z^{\prime} x}^{\text {mat }} n_{y^{\prime} y}^{\text {mat }} \\
& n_{x^{\prime} y}^{m a t} n_{z^{\prime} y}^{m a t} \\
& n_{x^{\prime} z}^{m a t} n_{z^{\prime} z}^{m a t} \\
& n_{x^{\prime} y}^{m a t} n_{y^{\prime} x}^{m a t}+n_{x^{\prime} x}^{m a t} n_{y^{\prime} y}^{m a t} \\
& n_{x^{\prime} x}^{m a t} n_{z^{\prime} y}^{m a t}+n_{x^{\prime} y}^{m a t} n_{z^{\prime} x}^{m a t} \\
& 2 n_{x^{\prime} y}^{m a t} n_{x^{\prime} z}^{m a t} \\
& 2 n_{y^{\prime} y}^{m a t} n_{y^{\prime} z}^{m a t} \\
& 2 n_{z^{\prime} y}^{\text {mat }} n_{z^{\prime} z}^{\text {mat }} \\
& n_{x^{\prime} y}^{m a t} n_{y^{\prime} z}^{m a t}+n_{x^{\prime} z}^{m a t} n_{y^{\prime} y}^{m a t} \\
& n_{y^{\prime} z}^{m a t} n_{z^{\prime} y}^{m a t}+n_{y^{\prime} y}^{m a t} n_{z^{\prime} z}^{m a t} \\
& n_{x^{\prime} y}^{m a t} n_{z^{\prime} z}^{m a t}+n_{x^{\prime} z}^{m a t} n_{z^{\prime} y}^{m a t} \\
& 2 n_{x^{\prime} x}^{m a t} n_{x^{\prime} z}^{m a t} \\
& 2 n_{y^{\prime} x}^{m a t} n_{y^{\prime} z}^{m a t} \\
& 2 n_{z^{\prime} x}^{m a t} n_{z^{\prime} z}^{m a t} \\
& n_{x^{\prime} x}^{m a t} n_{y^{\prime} z}^{m a t}+n_{y^{\prime} x}^{m a t} n_{x^{\prime} z}^{m a t} \\
& n_{y^{\prime} x}^{m a t} n_{z^{\prime} z}^{m a t}+n_{z^{\prime} x}^{m a t} n_{y^{\prime} z}^{m a t} \\
& \left.n_{x^{\prime} z}^{m a t} n_{z^{\prime} x}^{m a t}+n_{x^{\prime} x}^{m a t} n_{z^{\prime} z}^{m a t}\right)
\end{aligned}
$$


with $\mathbf{n}_{x^{\prime}}^{\text {mat }}=\left(n_{x^{\prime} x}^{\text {mat }}, n_{x^{\prime} y}^{\text {mat }}, n_{x^{\prime} z}^{\text {mat }}\right)^{\top} \in \mathfrak{R}^{3}, \mathbf{n}_{y^{\prime}}^{\text {mat }}=\left(n_{y^{\prime} x}^{\text {mat }}, n_{y^{\prime} y}^{\text {mat }}, n_{y^{\prime} z}^{\text {mat }}\right)^{\top} \in \mathfrak{R}^{3}$ and $\mathbf{n}_{z^{\prime}}^{\text {mat }}=\left(n_{z^{\prime} x}^{\text {mat }}, n_{z^{\prime} y}^{\text {mat }}, n_{z^{\prime} z}^{\text {mat }}\right)^{\top} \in \mathfrak{R}^{3}$ as the three normal vectors of the material local coordinate basis.

In order to derive a traction-based yield function for velocity discontinuities based on Eq. (7), the first step is the transformation of this yield function from the material local coordinate basis $\left(x^{\prime}-y^{\prime}-z^{\prime}\right)_{m a t}$ into the discontinuity local coordinate basis $(n-m-p)_{d i s}$, reading

$$
f_{m a t}\left(\sigma_{l o c}^{d i s}\right)=\sigma_{l o c}^{d i s} \mathbf{P}_{\sigma}^{d i s} \boldsymbol{\sigma}_{d i s}^{d i s}+\left(\frac{1}{2} \mathbf{F}_{\sigma}^{d i s,+\boldsymbol{\top}} \boldsymbol{\sigma}_{l o c}^{d i s}\right)^{2}-\left(1-\frac{1}{2} \mathbf{F}_{\sigma}^{d i s,-\boldsymbol{\top}} \boldsymbol{\sigma}_{l o c}^{d i s}\right)^{2} \leq 0, \quad \text { on } \Gamma_{d i s}
$$

where $\boldsymbol{\sigma}_{l o c}^{d i s}=\mathbf{R}_{\sigma}^{d i s} \boldsymbol{\sigma}$ denotes a local stress state at the discontinuity with respect to the discontinuity local coordinate basis. $\mathbf{R}_{\sigma}^{d i s}$ is the related transformation matrix equally defined as the matrix in Eq. (8), with $\mathbf{n}_{n}^{d i s}=\left(n_{n x}^{d i s}, n_{n y}^{d i s}, n_{n z}^{d i s}\right)^{\top} \in$ $\mathfrak{R}^{3}, \mathbf{n}_{m}^{d i s}=\left(n_{m x}^{d i s}, n_{m y}^{d i s}, n_{m z}^{d i s}\right)^{\top} \in \mathfrak{R}^{3}$ and $\mathbf{n}_{p}^{d i s}=\left(n_{p x}^{d i s}, n_{p y}^{d i s}, n_{p z}^{d i s}\right)^{\top} \in \mathfrak{R}^{3}$ as the normal vectors of the local coordinate basis $(n-m-p)_{d i s}$ (with $\mathbf{n}_{n}^{d i s}$ as the out of plane normal vector and $\mathbf{n}_{m}^{d i s}$ and $\mathbf{n}_{p}^{\text {dis }}$ as the in plane normal vectors). According to these definitions, $\boldsymbol{\sigma}_{l o c}^{m a t}\left(\right.$ Eq. (7)) and $\boldsymbol{\sigma}_{l o c}^{\text {dis }}$ (Eq. (9)) are connected as follows: $\boldsymbol{\sigma}_{l o c}^{m a t}=\mathbf{R}_{\sigma}^{m a t}\left(\mathbf{R}_{\sigma}^{\text {dis }}\right)^{-1} \boldsymbol{\sigma}_{l o c}^{\text {dis }}$. The yield function matrices in Eq. (9) can then be determined as

$$
\begin{aligned}
& \mathbf{P}_{\sigma}^{d i s}=\left(\mathbf{R}_{\sigma}^{m a t}\left(\mathbf{R}_{\sigma}^{d i s}\right)^{-1}\right)^{\top} \mathbf{P}_{\sigma}^{m a t}\left(\mathbf{R}_{\sigma}^{m a t}\left(\mathbf{R}_{\sigma}^{d i s}\right)^{-1}\right) \in \mathfrak{R}^{6 \times 6} \\
& \mathbf{F}_{\sigma}^{d i s,+}=\left(\mathbf{R}_{\sigma}^{m a t}\left(\mathbf{R}_{\sigma}^{d i s}\right)^{-1}\right)^{\top} \mathbf{F}_{\sigma}^{m a t,+} \in \mathfrak{R}^{6} \\
& \mathbf{F}_{\sigma}^{d i s,-}=\left(\mathbf{R}_{\sigma}^{m a t}\left(\mathbf{R}_{\sigma}^{d i s}\right)^{-1}\right)^{\top} \mathbf{F}_{\sigma}^{m a t,-} \in \mathfrak{R}^{6}
\end{aligned}
$$

Next, the local stress field at discontinuities $\boldsymbol{\sigma}_{l o c}^{d i s}$ is linked to the corresponding traction field $\mathbf{t}_{l o c}^{d i s}$ simply by

$$
\mathbf{t}_{l o c}^{d i s}=\mathbf{L}_{\sigma}^{d i s} \boldsymbol{\sigma}_{l o c}^{d i s}, \quad \text { on } \Gamma_{d i s}
$$

with

$$
\mathbf{L}_{\sigma}^{d i s}=\left(\begin{array}{llllll}
1 & 0 & 0 & 0 & 0 & 0 \\
0 & 0 & 0 & 1 & 0 & 0 \\
0 & 0 & 0 & 0 & 0 & 1
\end{array}\right) \in \Re^{3 \times 6}
$$

as the projection operator. Thereby, the local traction field is obtained by $\mathbf{t}_{l o c}^{d i s}=\mathbf{R}_{t}^{d i s} \mathbf{t}$, with the transformation matrix

$$
\mathbf{R}_{t}^{d i s}=\left(\begin{array}{ccc}
n_{n x}^{d i s} & n_{n y}^{d i s} & n_{n z}^{d i s} \\
n_{m x}^{d i s} & n_{m y}^{d i s} & n_{m z}^{d i s} \\
n_{p x}^{d i s} & n_{p y}^{d i s} & n_{p z}^{d i s}
\end{array}\right) \in \Re^{3 \times 3}
$$

According to Eq. (11), it is obvious that, from a critical stress state, the corresponding critical traction forces at a discontinuity can easily be evaluated. However, the reverse way, evaluating whether a traction force state is critical or not with respect to the stress-based yield function in Eq. (9), is not possible without further conditions, because the local traction field $\mathbf{t}_{l o c}^{d i s}$ only defines three stress components $\left(\sigma_{n n}^{d i s}, \sigma_{n m}^{d i s}, \sigma_{n p}^{d i s}\right)$ of the related local 3D stress state. For this reason, three additional constraints are needed to ensure that the finally derived traction-based yield function $f_{\text {dis }}(\mathbf{t}) \leq 0$ is only triggered for traction force states which in any case lead to plastic failure. This can be achieved by adding the following three constraints:

$$
\begin{aligned}
& \dot{\varepsilon}_{m m}^{d i s}=\Lambda_{m m}^{d i s}\left(\sigma_{l o c}^{d i s}\right)=\dot{\lambda}_{\dot{\varepsilon}}^{d i s} \partial f_{m a t}\left(\sigma_{l o c}^{d i s}\right) / \partial \sigma_{m m}^{d i s}=0 \\
& \dot{\varepsilon}_{p p}^{d i s}=\Lambda_{p p}^{d i s}\left(\sigma_{l o c}^{d i s}\right)=\dot{\lambda}_{\dot{\varepsilon}}^{d i s} \partial f_{m a t}\left(\sigma_{l o c}^{d i s}\right) / \partial \sigma_{p p}^{d i s}=0 \\
& \dot{\varepsilon}_{m p}^{d i s}=\Lambda_{m p}^{d i s}\left(\sigma_{l o c}^{d i s}\right)=\dot{\lambda}_{\dot{\varepsilon}}^{d i s} \partial f_{m a t}\left(\sigma_{l o c}^{d i s}\right) / \partial \sigma_{m p}^{d i s}=0
\end{aligned}
$$

enforcing the plastic strain-rate field $\dot{\boldsymbol{\varepsilon}}_{l o c}^{\text {dis }}$ to be localised with respect to the discontinuity $\Gamma_{d i s}\left((n-m-p)_{d i s}\right)$, as introduced by $\mathrm{Wu}$ and Cervera [51]. Thereby, $\Lambda_{m m}^{d i s}, \Lambda_{p p}^{d i s}$ and $\Lambda_{m p}^{d i s}$ represent the partial derivative expressions of the yield function Eq. (9) with respect to the three stress components $\sigma_{m m}^{d i s}, \sigma_{p p}^{d i s}$ and $\sigma_{m p}^{d i s}$, irrelevant to the interface plane of $\Gamma_{d i s}$. 
Substituting the stress components $\sigma_{n n}^{d i s}, \sigma_{n m}^{d i s}$ and $\sigma_{n p}^{d i s}$ in Eq. (14) with the local traction components $t_{n}^{d i s}, t_{m}^{d i s}$ and $t_{p}^{d i s}$ according to Eqs. (11) and (12), the remaining stress components can be expressed as functions $L$ of the local traction vector $\mathbf{t}_{l o c}^{d i s}$, reading

$$
\begin{gathered}
\sigma_{m m}^{d i s}=L_{m m}^{d i s}\left(\mathbf{t}_{l o c}^{d i s}\right) \\
\sigma_{p p}^{d i s}=L_{p p}^{d i s}\left(\mathbf{t}_{l o c}^{d i s}\right) \\
\sigma_{m p}^{d i s}=L_{m p}^{d i s}\left(\mathbf{t}_{l o c}^{d i s}\right)
\end{gathered}
$$

Now, each local traction force state $\mathbf{t}_{l o c}^{d i s}$ can be linked to a unique 3D local stress state $\sigma_{l o c}^{d i s}$, reading

$$
\sigma_{l o c}^{d i s}=\mathbf{L}_{t}^{d i s} \mathbf{t}_{l o c}^{d i s}, \quad \text { on } \Gamma_{d i s}
$$

where $\mathbf{L}_{t}^{\text {dis }} \in \mathfrak{R}^{6 \times 3}$ represents the projection operator determined from Eqs. (11) and (15) with respect to $\Gamma_{d i s}$.

Finally, by inserting Eq. (16) into Eq. (9), the stress-based yield function $f_{\text {mat }}\left(\sigma_{l o c}^{d i s}\right) \leq 0$ in $\Omega$ can be projected onto a traction-based yield function $f_{d i s}\left(\mathbf{t}_{l o c}^{d i s}\right) \leq 0$ locally on $\Gamma_{d i s}$, which reads

$$
\begin{aligned}
& f_{m a t}\left(\boldsymbol{\sigma}_{l o c}^{d i s}\right)=\sigma_{l o c}^{d i s \boldsymbol{\top}} \mathbf{P}_{\sigma}^{d i s} \boldsymbol{\sigma}_{d i s}^{d i s}+\left(\frac{1}{2} \mathbf{F}_{\sigma}^{d i s,+\boldsymbol{\top}} \boldsymbol{\sigma}_{l o c}^{d i s}\right)^{2}-\left(1-\frac{1}{2} \mathbf{F}_{\sigma}^{d i s,-\top} \boldsymbol{\sigma}_{l o c}^{d i s}\right)^{2} \\
& =\left(\mathbf{L}_{t}^{d i s} \mathbf{t}_{l o c}^{d i s}\right)^{\top} \mathbf{P}_{\sigma}^{d i s}\left(\mathbf{L}_{t}^{d i s} \mathbf{t}_{l o c}^{d i s}\right)+\left(\frac{1}{2} \mathbf{F}_{\sigma}^{d i s,+\boldsymbol{\top}}\left(\mathbf{L}_{t}^{d i s} \mathbf{t}_{l o c}^{d i s}\right)\right)^{2}-\left(1-\frac{1}{2} \mathbf{F}_{\sigma}^{d i s,-\boldsymbol{\top}}\left(\mathbf{L}_{t}^{d i s} \mathbf{t}_{l o c}^{d i s}\right)\right)^{2} \\
& =\mathbf{t}_{l o c}^{d i s \top} \mathbf{P}_{t}^{d i s} \mathbf{t}_{l o c}^{d i s}+\left(\frac{1}{2} \mathbf{F}_{t}^{d i s,+\top} \mathbf{t}_{l o c}^{d i s}\right)^{2}-\left(1-\frac{1}{2} \mathbf{F}_{t}^{d i s,-\top} \mathbf{t}_{l o c}^{d i s}\right)^{2} \quad=f_{d i s}\left(\mathbf{t}_{l o c}^{d i s}\right)
\end{aligned}
$$

where $\mathbf{P}_{t}^{d i s}=\mathbf{L}_{t}^{d i s}{ }^{\text {P }}{ }_{\sigma}^{d i s} \mathbf{L}_{t}^{d i s} \in \mathfrak{R}^{3 \times 3}, \mathbf{F}_{t}^{d i s,+}=\mathbf{L}_{t}^{d i s \boldsymbol{T}} \mathbf{F}_{\sigma}^{d i s,+} \in \mathfrak{R}^{3}$ and $\mathbf{F}_{t}^{d i s,-}=\mathbf{L}_{t}^{d i s \boldsymbol{T}} \mathbf{F}_{\sigma}^{d i s,-} \in \mathfrak{R}^{3}$ are the matrices containing strength parameters for the traction-based yield function.

\subsection{Numerical verification}

In the following, the presented transition (Eq. (17)) from a stress-based yield function into a traction-based yield function is numerically verified. Assuming the material local coordinate basis as identical to the global coordinate basis, arbitrary Tsai-Wu strength parameters for the stress-based yield function (Eq. (7)) are chosen as

$$
\mathbf{F}_{\sigma}^{m a t}=\left(\begin{array}{c}
0.0750 \\
0.7500 \\
0.1905 \\
0 \\
0 \\
0
\end{array}\right) \quad \mathbf{P}_{\sigma}^{\text {mat }}=\left(\begin{array}{cccccc}
0.0250 & 0 & 0 & 0 & 0 & 0 \\
0 & 0.2500 & 0 & 0 & 0 & 0 \\
0 & 0 & 0.0476 & 0 & 0 & 0 \\
0 & 0 & 0 & 0.1111 & 0 & 0 \\
0 & 0 & 0 & 0 & 0.2500 & 0 \\
0 & 0 & 0 & 0 & 0 & 0.0400
\end{array}\right)
$$

Then, a set of failure stress states $\sigma^{i}\left(i \in\left\{1, \cdots, n_{f}\right\}\right)$ with respect to the global coordinate basis $(x-y-z)$ can be determined by inserting the parameters in Eq. (18) into the general form of the yield function in Eq. (7), where $n_{f}$ represents the number of selected failure stress states.

Considering two different and arbitrarily selected planes (discontinuities) $\Gamma_{1}$ and $\Gamma_{2}$ defined by their normal vectors

$$
\begin{array}{ll}
\mathbf{n}_{1}=(0.1826,0.9129,0.3651)^{\top} & \text { for } \Gamma_{1} \\
\mathbf{n}_{2}=(-0.8018,0.5345,-0.2673)^{\top} & \text { for } \Gamma_{2}
\end{array}
$$

two sets of local traction force states $\mathbf{t}_{\sigma, 1}^{d i s, i}$ and $\mathbf{t}_{\sigma, 2}^{d i s, i}\left(i \in\left\{1, \cdots, n_{\sigma}\right\}\right)$ can be determined as

$$
\mathbf{t}_{\sigma, j}^{d i s, i}=\mathbf{L}_{\sigma}^{d i s} \mathbf{R}_{\sigma, j}^{d i s} \boldsymbol{\sigma}^{i} \quad \forall i \in\left\{1, \cdots, n_{\sigma}\right\} \text { and } j \in\{1,2\}
$$

with respect to their local coordinate bases $(n-m-p)_{1}$ and $(n-m-p)_{2}$, respectively. In Eq. (20), $\mathbf{L}_{\sigma}^{d i s}$ denotes the operator projecting the stress state into the traction force state defined in Eq. (12), and $\mathbf{R}_{\sigma, 1}^{d i s}$ and $\mathbf{R}_{\sigma, 2}^{d i s}$ are the 


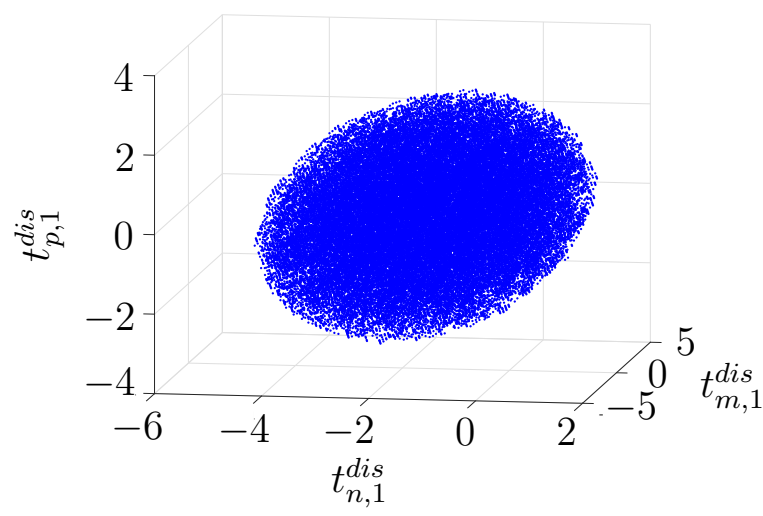

(a)

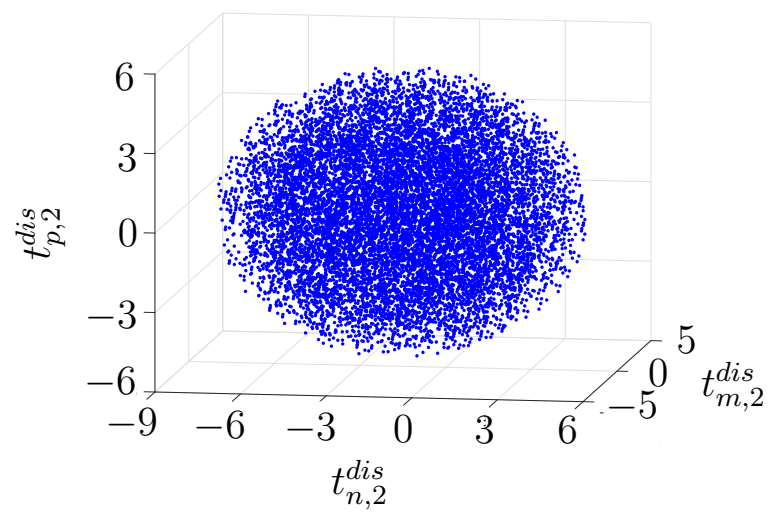

(b)

Figure 1: Local traction point clouds determined by projecting the set of global failure stress state $\boldsymbol{\sigma}^{i}$ into two sets of local traction states (a) $\mathbf{t}_{l o c, 1}^{\text {dis, }}$ on the plane $\Gamma_{1}$ with respect to $(n-m-p)_{1}$ and (b) $\mathbf{t}_{l o c, 2}^{d i s, i}$ on the plane $\Gamma_{2}$ with respect to $(n-m-p)_{2}$ where $\left(i \in\left\{1, \cdots, n_{f}\right\}\right)$.

transformation matrices similar to Eq. (8) based on the axial normal vectors $\mathbf{n}_{n, 1}=(0.1826,0.9129,0.3651)^{\top}, \mathbf{n}_{m, 1}=$ $(-0.9806,0.1961,0.0000)^{\top}, \mathbf{n}_{p, 1}=(-0.0716,-0.3581,0.9309)^{\top}$ for $(n-m-p)_{1}$ and $\mathbf{n}_{n, 2}=(-0.8018,0.5345,-0.2673)^{\top}$, $\mathbf{n}_{m, 2}=(0.5547,0.8321,0.0000)^{\top}, \mathbf{n}_{p, 2}=(0.2224,-0.1483,-0.9636)^{\top}$ for $(n-m-p)_{2}$, respectively.

Although all failure stress states $\sigma^{i}$ naturally lie on a continuous surface, as defined through the stress-based yield function in Eq. (7), the corresponding traction force failure stress states $\mathbf{t}_{\sigma, i}^{d i s, i}$ and $\mathbf{t}_{\sigma, 2}^{\text {dis, }}$, obtained through Eq. (20) are not necessarily located on such. They rather represent point clouds whose shapes heavily depend on the orientation of the planes $\Gamma_{1}$ and $\Gamma_{2}$, as illustrated in Figure 1. However, a clear ellipsoidal outer envelope can be observed, meaning that every traction force state lying outside this envelope is for sure related to a failure stress state according to Eq. (7).

According to Eq. (17), the stress-based yield function using the strength parameters in Eq. (18) can be projected into the traction-based yield functions with respect to the local coordinate basis $(n-m-p)_{1}$ and $(n-m-p)_{2}$ for which the strength parameters are determined as

$$
\begin{aligned}
& \mathbf{F}_{t, 1}^{d i s}=\left(\begin{array}{c}
0.4584 \\
0.1290 \\
-0.1012
\end{array}\right) \quad \mathbf{P}_{t, 1}^{d i s}=\left(\begin{array}{ccc}
0.1515 & 0.0424 & -0.0485 \\
0.0424 & 0.0780 & -0.0111 \\
-0.0485 & -0.0111 & 0.1488
\end{array}\right) \quad \text { for } \Gamma_{1} \\
& \mathbf{F}_{t, 2}^{d i s}=\left(\begin{array}{c}
0.0899 \\
0.0791 \\
-0.0020
\end{array}\right) \quad \mathbf{P}_{t, 2}^{d i s}=\left(\begin{array}{ccc}
0.0296 & 0.0260 & -0.0037 \\
0.0260 & 0.0782 & -0.0033 \\
-0.0037 & -0.0033 & 0.0356
\end{array}\right) \text { for } \Gamma_{2}
\end{aligned}
$$

Inserting these strength parameters into the general form of the traction-based yield function in Eq. (17c) leads to yield functions for the two planes $\Gamma_{1}$ and $\Gamma_{2}$, respectively.

For each plane, a set of traction force failure states $\mathbf{t}_{t, j}^{d i s, i}\left(i \in\left\{1, \cdots, n_{t}\right\}\right.$ and $\left.j \in\{1, \cdots, 2\}\right)$ is evaluated and plotted in Figure 2 as red point cloud $\left(n_{t}\right.$ is the number of selected failure traction states based on the traction-based yield function), where the blue point cloud is identical to Figure 1. It can be seen that, for each example plane, the red point cloud spans a continuous surface identical to the outer envelope of the blue point cloud. This proves that the presented traction-based yield function in Eq. (17c) represents a suitable criterion to indicate wether a traction force state at a discontinuity should exhibit plastic deformation or not. Of course, there are plenty of traction force states which could lead to failure but do not violate the traction-based yield function. In these cases plastic deformation takes place in the adjacent solid finite elements, and the upper bound theorem itself will never be violated. Thus, to exploit the performance of discontinuities in this kind of formulation, they should be aligned according to planes where strain localisation takes place. This requires an adaptive implementation, which will be presented and discussed in Section 6. 


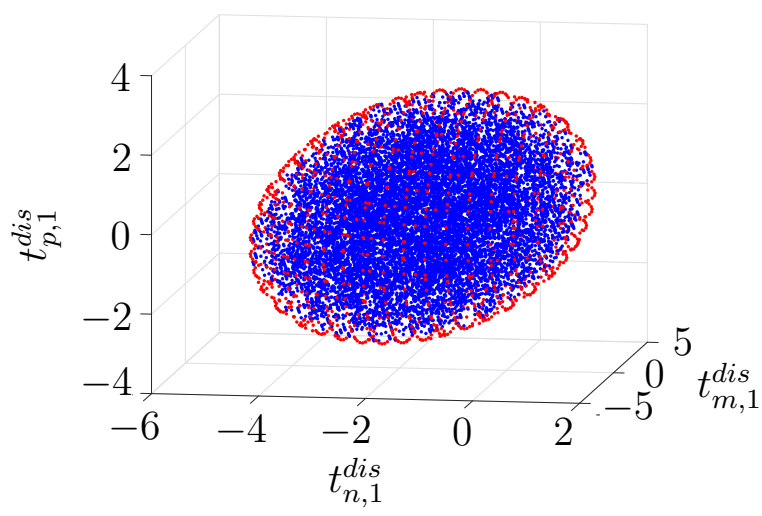

(a)

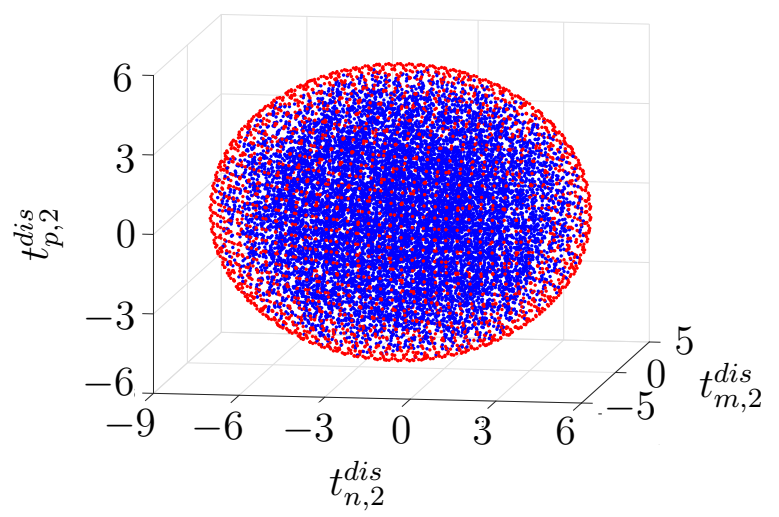

(b)

Figure 2: Verification of the projection in Eq. (17) by means of two example planes (a) $\Gamma_{1}$ and (b) $\Gamma_{2}$, where the blue point clouds indicate all possible traction states, $\mathbf{t}_{\sigma, 1}^{d i s, i}$ and $\mathbf{t}_{\sigma, 2}^{d i s, i}$, related to the yield stress states $\boldsymbol{\sigma}^{i}$ determined by the stress-based yield function with the parameters in Eq. (18) $\left(i \in\left\{1, \cdots, n_{\sigma}\right\}\right)$. The red point clouds indicate the yield traction states $\mathbf{t}_{t, 1}^{d i s, j}$ and $\mathbf{t}_{t, 2}^{d i s, j}$ according to the traction-based yield function with the parameters in Eq. (21) $\left(j \in\left\{1, \cdots, n_{t}\right\}\right)$.

\section{Associated plastic flow rule}

In both the primal and dual form of the upper bound problem in Eqs. (3) and (5), all nonlinear constraints are related to the yield functions and their associated plastic flow rule. As stated by Makrodimopoulos and Martin [21] and Ciria et al. [7], for 3D problems, most commonly used failure criteria are smooth and can be formulated into secondorder cone constraints (e.g. the von Mises failure criterion, the Drucker-Prager failure criterion and the Tsai-Wu failure criterion), thus, the nonlinear optimisation problems in Eqs. (3) and (5) can be solved efficiently by using SOCP. The widely-used 3D Mohr-Coulomb failure criterion is non-smooth and, thus, cannot be formulated into a second-order cone. However, using semidefinite programming instead of second-order cone programming, as introduced by Martin and Makrodimopoulos [25] and Krabbenhøft et al. [52], this problem can be overcome. In the following, only formulations of smooth yield functions and their associated plastic flow rule are considered and their reformulation as second-order cone constraints is described.

\section{Associated plastic flow rule in the continuum}

In the continuum $\Omega$ the plastic flow is measured by the strain-rate field and the associated plastic flow rule reads

$$
\dot{\varepsilon}_{l o c}^{m a t}=\dot{\lambda}_{\dot{\varepsilon}}^{m a t} \partial f_{m a t}\left(\sigma_{l o c}^{m a t}\right) / \partial \sigma_{l o c}^{m a t}
$$

where $\dot{\boldsymbol{\varepsilon}}_{l o c}^{\text {mat }}$ represents the strain-rate field with respect to the material local coordinate basis $\left(x^{\prime}-y^{\prime}-z^{\prime}\right)_{m a t}$ and $\dot{\lambda}_{l o c}^{\text {mat }}$ is the corresponding plastic multiplier rate.

If the stress-based yield functions can be formulated according to Eq. (7) and the parametric matrix $\mathbf{P}_{\sigma}^{\text {mat }}$ is either positive definite or positive semidefinite, so that it can be decomposed as $\mathbf{P}_{\sigma}^{\text {mat }}=\mathbf{D}_{\sigma}^{\text {mat }} \mathbf{D}_{\sigma}^{\text {mat }}$ with $\mathbf{D}_{\sigma}^{\text {mat }} \in \mathfrak{R}^{6 \times 6}$, the yield function $f_{\text {mat }}\left(\sigma_{l o c}^{\text {mat }}\right) \leq 0$ can be reformulated as

$$
\begin{aligned}
& \mathbf{s}_{\sigma}^{m a t}=\mathbf{a}_{\sigma}^{m a t}+\mathbf{B}_{\sigma}^{m a t} \sigma_{l o c}^{m a t} \\
& \mathbf{s}_{\sigma}^{m a t} \in \mathscr{C}
\end{aligned}
$$

where $\mathbf{s}_{\sigma}^{\text {mat }} \in \mathfrak{R}^{8}$ is the second-order cone expression of the stress-based yield function Eq. (7), $\mathscr{C}$ refers to the quadratic cone, and the parametric matrices are composed as follows

$$
\mathbf{a}_{\sigma}^{m a t}=\left(\begin{array}{l}
1 \\
\mathbf{0}
\end{array}\right) \in \mathfrak{R}^{8} \quad \text { and } \quad \mathbf{B}_{\sigma}^{m a t}=\left(\begin{array}{c}
-\frac{1}{2} \mathbf{F}_{\sigma}^{m a t,-\boldsymbol{T}} \\
\mathbf{D}_{\sigma}^{m a t} \\
\frac{1}{2} \mathbf{F}_{\sigma}^{m a t,+\boldsymbol{\top}}
\end{array}\right) \in \mathfrak{R}^{8 \times 6}
$$


according to the definition of second-order cones (Eq. (A.3)).

As applied by Makrodimopoulos and Martin [21] and Li et al. [49], if the yield function can be formulated as second-order cone constraint (Eq. (23)), the corresponding associated plastic flow rule Eq. (22) can be formulated as

$$
\begin{aligned}
& \dot{\boldsymbol{\varepsilon}}_{l o c}^{m a t}=-\mathbf{B}_{\sigma}^{m a t}{ }^{m} \dot{\mathbf{e}}_{\dot{\varepsilon}}^{m a t} \\
& \dot{\mathbf{e}}_{\dot{\varepsilon}}^{m a t} \in \mathscr{C}^{*}
\end{aligned}
$$

where $\dot{\mathbf{e}}_{\dot{\varepsilon}}^{\text {mat }} \in \mathfrak{R}^{8}$ represents the second-order cone expression of the associated plastic flow rule and $\mathscr{C}^{*}$ is the dual cone of the primal quadratic cone $\mathscr{C}$ of Eq. (23).

\section{Associated plastic flow rule at velocity discontinuities}

As introduced in Section 2, velocity-jumps $\Delta \dot{\mathbf{u}}$ are employed in the primal upper bound problem Eq. (3) as additional degrees of freedom beside the strain-rate field $\dot{\boldsymbol{\varepsilon}}$ measuring the localised plastic flow. At velocity discontinuities $\Gamma_{\text {dis }}$ plastic flow occurs when the traction-based yield function according to Eq. $(17 \mathrm{c})$ is violated. Then, a velocity jump field between the surfaces $\Gamma_{d i s}^{+}$and $\Gamma_{d i s}^{-}$appears, given by

$$
\Delta \dot{\mathbf{u}}_{l o c}^{d i s}=\dot{\lambda}_{l o c}^{d i s} \partial f_{d i s}\left(\mathbf{t}_{l o c}^{d i s}\right) / \partial \mathbf{t}_{l o c}^{d i s}
$$

where $\Delta \dot{\mathbf{u}}_{l o c}^{d i s}=\mathbf{R}_{\Delta \dot{u}}^{d i s} \Delta \dot{\mathbf{u}}$ is the local velocity discontinuity field with respect to $(n-m-p)_{d i s}, \mathbf{R}_{\Delta u}^{d i s}=\mathbf{R}_{t}^{d i s}$ is the transformation matrix with similar definition as in Eq. (13), and $\dot{\lambda}_{\text {loc }}^{\text {dis }}$ denotes the corresponding plastic multiplier rate.

As indicated in Eq. (17), the traction-based yield function exhibits a similar structure as the stress-based yield function and, thus, can also be formulated as second-order cone constraint, reading

$$
\begin{aligned}
& \mathbf{s}_{t}^{d i s}=\mathbf{a}_{t}^{d i s}+\mathbf{B}_{t}^{d i s} \mathbf{t}_{l o c}^{d i s} \\
& \mathbf{s}_{t}^{d i s} \in \mathscr{C}
\end{aligned}
$$

where $\mathbf{s}_{t}^{d i s} \in \mathfrak{R}^{5}$ is the second-order cone expression of the traction-based yield function Eq. (17c) and the parametric matrices are composed as follows

$$
\mathbf{a}_{t}^{d i s}=\left(\begin{array}{l}
1 \\
\mathbf{0}
\end{array}\right) \in \mathfrak{R}^{5} \quad \text { and } \quad \mathbf{B}_{t}^{d i s}=\left(\begin{array}{c}
-\frac{1}{2} \mathbf{F}_{t}^{d i s,-\boldsymbol{T}} \\
\mathbf{D}_{t}^{d i s} \\
\frac{1}{2} \mathbf{F}_{t}^{d i s,+\boldsymbol{\top}}
\end{array}\right) \in \mathfrak{R}^{5 \times 3}
$$

with $\mathbf{D}_{t}^{d i s} \in \mathfrak{R}^{3 \times 3}$ as the decomposed matrix of $\mathbf{P}_{t}^{d i s}=\mathbf{D}_{t}^{d i s} \mathbf{\mathbf { D } _ { t }}$ dis.

Furthermore, similar to Eq. (25), the associated plastic flow rule at $\Gamma_{d i s}$ can be formulated as the dual cone constraint of Eq. (27), reading

$$
\begin{aligned}
\Delta \dot{\mathbf{u}}_{l o c}^{d i s} & =-\mathbf{B}_{t}^{d i s \top} \mathbf{e}_{\Delta \dot{u}}^{d i s} \\
\dot{\mathbf{e}}_{\Delta \dot{u}}^{d i s} & \in \mathscr{C}^{*}
\end{aligned}
$$

$$
\text { where } \dot{\mathbf{e}}_{\Delta \dot{u}}^{d i s} \in \mathfrak{R}^{5} \text { is the second-order cone expression of Eq. (26). }
$$

\section{Finite-element-based upper bound formulation}

In this work, 10-noded simplex strain tetrahedral elements are used for discretisation, which are capable of providing more accurate upper bound results compared to the often used 4-noded (constant strain rate) elements $[21,25]$. In each element $e l$, the strain-rate field $\dot{\varepsilon}$ is obtained through interpolation between nodal strain-rate vectors $\mathbf{q}_{\dot{\varepsilon}}^{e l, i}=\left(q_{\dot{\varepsilon}, x x}^{e l, i}, q_{\dot{\varepsilon}, y y}^{e l, i}, q_{\dot{\varepsilon}, z z}^{e l, i}, q_{\dot{\varepsilon}, x y}^{e l, i}, q_{\dot{\varepsilon}, y z}^{e l, i}, q_{\dot{\varepsilon}, x z}^{e l, i}\right)^{\top} \in \mathfrak{R}^{6}$ at 4 evaluation nodes (4 vertices) using 3D linear interpolation functions $N_{\dot{\varepsilon}}^{e l, i}(\mathbf{x})(\forall e l \in\{1, \cdots, U E\}$ and $i \in\{1, \cdots, 4\})$ and the velocity field $\dot{\mathbf{u}}$ is obtained through interpolation between nodal velocity vectors $\mathbf{q}_{i}^{e l, j}=\left(q_{\dot{u}, x}^{e l, j}, q_{\dot{u}, y}^{e l, j}, q_{u, z}^{e l, j}\right)^{\top} \in \mathfrak{R}^{3}$ at 10 evaluation nodes (4 vertices and 6 edge middle nodes) using 3D quadratic interpolation functions $N_{\dot{u}}^{e l, j}(\mathbf{x})(\forall e l \in\{1, \cdots, U E\}$ and $j \in\{1, \cdots, 10\})$ with $U E$ as the total number of 
elements in the upper bound problem. Here, $\mathbf{x}=\left(x_{x}, x_{y}, x_{z}\right)^{\top} \in \mathfrak{R}^{3}$ represents an arbitrary position vector inside each element. In the discretised structure, the strain-rate field $\dot{\varepsilon}$ is piecewise linear within each element and allowed to be discontinuous between elements, thus, each element has its own internal strain-rate evaluation nodes but adjacent nodes from different elements can share the same coordinate.

Consequently, each boundary surface of an element $b e$ is triangular with 6 velocity evaluation nodes (3 vertices and 3 edge middle nodes), and the velocity field $\dot{\mathbf{u}}$ is obtained through interpolation between nodal velocity vectors $\mathbf{q}_{\dot{u}}^{b e, k}$ using 2D quadratic interpolation functions $\bar{N}_{\dot{u}}^{b e, k}(\mathbf{x})(\forall b e \in\{1, \cdots, U B E\}$ and $k \in\{1, \cdots, 6\})$ with $U B E$ as the total number of 6-noded boundary elements on $\Gamma$.

As mentioned above, velocity jumps $\Delta \dot{\mathbf{u}}$ are only allowed at prescribed discontinuities $\Gamma_{d i s}$, where each surface pair $\Gamma_{d i s}^{+}$and $\Gamma_{d i s}^{-}$has its own set of internal velocity evaluation nodes with different nodal velocity vectors $\mathbf{q}_{\dot{u}}^{d e+, i}$ and $\mathbf{q}_{\dot{u}}^{d e-, j}(\forall d e \in\{1, \cdots, U D E\}$ and $i, j \in\{1, \cdots, 6\})$. Each triangular surface at a discontinuity de, located on the interface part $\Gamma_{d i s}^{d e} \subset \Gamma_{d i s}$, has 6 velocity evaluation nodes with corresponding 2D quadratic interpolation functions $\bar{N}_{\dot{u}}^{d e, i}(\mathbf{x})$. Here $U D E$ represents the total number of velocity discontinuity elements on $\Gamma_{d i s}$.

\subsection{Primal formulations}

The discretised form of each constraint in the primal upper bound problem (Eq. (3)) is introduced in the following.

\section{Compatibility within elements}

Based on the assumptions of small strains and straight element edges, all changes in geometry of the considered structure during collapse are negligible, and the discretised form of the first constraint in Eq. (3) can be written as

$$
\sum_{i=1}^{4} N_{\dot{\varepsilon}}^{e l, i}(\mathbf{x}) \mathbf{q}_{\dot{\varepsilon}}^{e l, i}=\mathbf{L}_{\dot{\varepsilon}} \sum_{j=1}^{10} N_{\dot{u}}^{e l, j}(\mathbf{x}) \mathbf{q}_{\dot{u}}^{e l, j}, \quad \forall e l \in\{1, \cdots, U E\}
$$

enforcing compatibility between the velocity field and the strain-rate field within each solid element $\Omega^{e l} \subset \Omega$.

\section{Compatibility across velocity discontinuities}

Since the strain-rate field $\dot{\boldsymbol{\varepsilon}}$ is linear within each solid element $\Omega^{e l}$, to guarantee consistency of plastic flow, it is straightforward to enforce the velocity jump $\Delta \dot{\mathbf{u}}$ to be linear across each velocity discontinuity $\Gamma_{d i s}^{d e}$, where $\Delta \dot{\mathbf{u}}$ is obtained through interpolation between the nodal velocity jumps $\mathbf{q}_{\Delta \dot{u}}^{d e, i}=\left(q_{\Delta \dot{u}, x}^{d e, i}, q_{\Delta \dot{u}, y}^{d e, i}, q_{\Delta \dot{u}, z}^{d e, i}\right)^{\top} \in \mathfrak{R}^{3}$ at 3 evaluation nodes (3 vertices) using 2D linear interpolation functions $\bar{N}_{\Delta \dot{u}}^{d e, i}(\mathbf{x})(\forall d e \in\{1, \cdots, U D E\}$ and $i \in\{1, \cdots, 3\})$.

Then, the second constraint in Eq. (3) reads in discretised form as follows:

$$
\sum_{i=1}^{3} \bar{N}_{\Delta \dot{u}}^{d e, i}(\mathbf{x}) \mathbf{q}_{\Delta \dot{u}}^{d e, i}=\sum_{j=1}^{6} \mathbf{L}_{\Delta \dot{u}} \bar{N}_{\dot{u}}^{d e, j}(\mathbf{x}) \mathbf{q}_{\dot{u}}^{d e, j}, \quad \forall d e \in\{1, \cdots, U D E\}
$$

with $\mathbf{L}_{\Delta \dot{u}}$ as defined in Eq. (4) and $\mathbf{q}_{\dot{u}}^{d e, j}=\left(\mathbf{q}_{\dot{u}}^{d e+, j}, \mathbf{q}_{\dot{u}}^{d e-, j}\right)^{\top} \in \mathfrak{R}^{3 \times 6}$ is a vector containing each pair of nodal velocities across the discontinuity $\Gamma_{d i s}$. Additional constraints are applied reading

$$
\begin{aligned}
\mathbf{L}_{\Delta \dot{u}} \mathbf{q}_{\dot{u}}^{d e, 4} & =\frac{1}{2}\left(\mathbf{L}_{\Delta \dot{u}} \mathbf{q}_{\dot{u}}^{d e, 1}+\mathbf{L}_{\Delta \dot{u}} \mathbf{q}_{\dot{u}}^{d e, 2}\right) \\
\mathbf{L}_{\Delta \dot{u}} \mathbf{q}_{\dot{u}}^{d e, 5} & =\frac{1}{2}\left(\mathbf{L}_{\Delta \dot{u}} \mathbf{q}_{\dot{u}}^{d e, 2}+\mathbf{L}_{\Delta \dot{u}} \mathbf{q}_{\dot{u}}^{d e, 3}\right), \quad \forall d e \in\{1, \cdots, U D E\} \\
\mathbf{L}_{\Delta \dot{u}} \mathbf{q}_{\dot{u}}^{d e, 6} & =\frac{1}{2}\left(\mathbf{L}_{\Delta \dot{u}} \mathbf{q}_{\dot{u}}^{d e, 1}+\mathbf{L}_{\Delta \dot{u}} \mathbf{q}_{\dot{u}}^{d e, 3}\right)
\end{aligned}
$$

in order to enforce a linear velocity jump distribution across each discontinuity. 
Kinematic boundary conditions

The kinematic boundary conditions are applied as

$$
\mathbf{q}_{\dot{u}}^{b e, i}=\mathbf{q}_{\dot{u}_{b c}}^{b e, i}, \quad \forall b e \in\{1, \cdots, U B E\} \text { and } i \in\{1, \cdots, 6\}
$$

where $\mathbf{q}_{\dot{u}_{b c}, i}^{b, i}$ is the prescribed nodal velocity vector at each velocity evaluation node on the boundary surface $\Gamma$.

\section{Associated plastic flow rule}

In each solid element $\Omega^{e l}$, the associated plastic flow rule is enforced at each strain-rate evaluation node, using the discretised form derived from Eq. (25), reading

$$
\begin{aligned}
& \mathbf{R}_{\dot{\varepsilon}}^{e l} \mathbf{q}_{\dot{\varepsilon}}^{e l, i}=-\mathbf{B}_{\sigma}^{e l \boldsymbol{\top}} \dot{\mathbf{e}}_{\dot{\varepsilon}}^{e l, i} \\
& \dot{\mathbf{e}}_{\dot{\varepsilon}}^{e l, i} \in \mathscr{C}^{*}
\end{aligned}, \quad \forall e l \in\{1, \cdots, U E\} \text { and } i \in\{1, \cdots, 4\}
$$

in which $\mathbf{q}_{\dot{\varepsilon}, l o c}^{e l, i}$ is the local nodal strain-rate vector determined from the transformation matrix $\mathbf{R}_{\dot{\varepsilon}}^{e l}$ with similar shape as Eq. (8) based on the axial normal vectors $\mathbf{n}_{x^{\prime}}^{e l}, \mathbf{n}_{y^{\prime}}^{e l}, \mathbf{n}_{z^{\prime}}^{e l}$ of the material local coordinate basis $\left(x^{\prime}-y^{\prime}-z^{\prime}\right)_{m a t}^{e l}$ of the element, $\dot{\mathbf{e}}_{\dot{\varepsilon}}^{e l, i} \in \mathfrak{R}^{8}$ is the nodal vector for the associated plastic flow rule Eq. (22) in the second-order cone expression and $\mathbf{B}_{\sigma}^{e l} \in \mathfrak{R}^{8 \times 6}$ is the parametric matrix defined in Eq. (24). Note that, in this paper the strength behaviours are considered as constant within one element.

Similarly, across each velocity discontinuity $\Gamma_{d i s}^{d e}$, the discretised associated plastic flow rule is derived from Eq. (29), reading

$$
\begin{aligned}
& \mathbf{R}_{\Delta \dot{u}}^{d e} \mathbf{q}_{\Delta \dot{u}}^{d e, i}=-\mathbf{B}_{t}^{d e \boldsymbol{\top}} \dot{\mathbf{e}}_{\Delta \dot{u}}^{d e, i} \\
& \dot{\mathbf{e}}_{\Delta \dot{u}}^{d e, i} \in \mathscr{C}^{*}
\end{aligned}, \quad \forall d e \in\{1, \cdots, U D E\} \text { and } i \in\{1, \cdots, 3\}
$$

where $\mathbf{q}_{\Delta \dot{u}, l o c}^{d e, i} \in \mathfrak{R}^{3}$ is the local nodal velocity-jump vector, $\mathbf{R}_{\Delta \dot{u}}^{d e}$ is the transformation matrix, defined similar as in Eq. (13), from the global coordinate basis $(x-y-z)$ into the local coordinate basis $(n-m-p)_{d i s}^{d e}$ for $\Gamma_{d i s}^{d e}$, $\dot{\mathbf{e}}_{\Delta u}^{d e}$ is the nodal second-order expression of the associated plastic flow rule Eq. (26) and $\mathbf{B}_{t}^{\text {de }} \in \mathfrak{R}^{5 \times 3}$ is the parametric matrix defined in Eq. (29).

\section{Internal dissipation rate}

Inserting Eqs. (30) and (31), the internal plastic dissipation rate Eq. (2) can be formulated numerically as

$$
\begin{aligned}
W_{i n t}= & \sum_{e l=1}^{U E} \int_{\Omega^{e l}} \dot{\boldsymbol{\varepsilon}}^{e l \boldsymbol{\top}} \boldsymbol{\sigma}^{e l} \mathrm{~d} V+\sum_{d e=1}^{U D E} \int_{\Gamma_{d i s}^{d e}} \Delta \dot{\mathbf{u}}_{l o c}^{d e \top} \mathbf{t}_{l o c}^{d e} \mathrm{~d} A \\
= & \sum_{e l=1}^{U E} \int_{\Omega^{e l}}\left(\mathbf{L}_{\dot{\varepsilon}} \sum_{i=1}^{10} N_{\dot{u}}^{e l, i}(\mathbf{x}) \mathbf{q}_{\dot{u}}^{e l, i}\right)^{\top}\left(\sum_{j=1}^{4} N_{\dot{\varepsilon}}^{e l, j}(\mathbf{x}) \mathbf{q}_{\sigma}^{e l, j}\right) \mathrm{d} V \\
& +\sum_{d e=1}^{U D E} \int_{\Gamma_{d i s}^{d e}}\left(\mathbf{L}_{\Delta \dot{u}} \sum_{i=1}^{6} \bar{N}_{\dot{u}}^{d e, i}(\mathbf{x}) \mathbf{q}_{\dot{u}}^{d e, i}\right)^{\top}\left(\sum_{j=1}^{3} \bar{N}_{\Delta \dot{u}}^{d e, j}(\mathbf{x}) \hat{\mathbf{q}}_{t}^{d e, j}\right) \mathrm{d} A \\
= & \sum_{e l=1}^{U E} \sum_{i=1}^{10} \sum_{j=1}^{4} \mathbf{q}_{\dot{u}}^{e l, i \boldsymbol{\top}} \mathbf{G}_{\dot{\varepsilon}}^{e l, i, j} \hat{\mathbf{q}}_{\sigma}^{e l, j}+\sum_{d e=1}^{U D E} \sum_{i=1}^{6} \sum_{j=1}^{3} \mathbf{q}_{\dot{u}}^{d e, i \boldsymbol{\top}} \overline{\mathbf{G}}_{\Delta \dot{u}}^{d e, i, j} \hat{\mathbf{q}}_{t}^{d e, j}
\end{aligned}
$$

where the numerical integration operators over each solid element $\Omega^{e l}$ and discontinuity $\Gamma_{d i s}^{d e}$ are defined as

$$
\begin{aligned}
\mathbf{G}_{\dot{\varepsilon}}^{e l, i, j} & =\int_{\Omega^{e l}} \mathbf{L}_{\dot{\varepsilon}}^{\top} N_{\dot{u}}^{e l, i}(\mathbf{x}) N_{\dot{\varepsilon}}^{e l, j}(\mathbf{x}) \mathrm{d} V \in \Re^{3 \times 6} \\
\overline{\mathbf{G}}_{\Delta \dot{u}}^{d e, k, l} & =\int_{\Gamma_{d i s}^{d e}} \mathbf{L}_{\Delta \dot{u}}^{\top} \bar{N}_{\dot{u}}^{d e, k}(\mathbf{x}) \bar{N}_{\Delta \dot{u}}^{d e, l}(\mathbf{x}) \mathrm{d} A \in \mathfrak{R}^{6 \times 3}
\end{aligned}
$$


with $\forall e l \in\{1, \cdots, U E\}, \forall d e \in\{1, \cdots, U D E\}, i \in\{1, \cdots, 10\}, j \in\{1, \cdots, 4\}, k \in\{1, \cdots, 6\}$ and $l \in\{1, \cdots, 3\}$.

As stated by Makrodimopoulos [53], since the upper bound problem is based on the kinematic theorem the stress field $\sigma^{e l}$ in each solid element $\Omega^{e l}$ and the traction field $\mathbf{t}_{l o c}^{d e}$ at each discontinuity $\Gamma_{d i s}^{d e}$ can not be numerically interpolated. Instead, nodal weighted-average stress and traction vectors are obtained, reading

$$
\begin{array}{rlrl}
\hat{\mathbf{q}}_{\sigma}^{e l, i} & =\int_{\Omega^{e l}} N_{\dot{\varepsilon}}^{e l, i}(\mathbf{x}) \sigma^{e l} \mathrm{~d} V / \int_{\Omega^{e l}} N_{\dot{\varepsilon}}^{e l, i}(\mathbf{x}) \mathrm{d} V, & & \forall e l \in\{1, \cdots, U E\} \text { and } i \in\{1, \cdots, 4\} \\
\hat{\mathbf{q}}_{t, l o c}^{d e, j} & =\int_{\Gamma_{d i s}^{d e}} \bar{N}_{\Delta \dot{u}}^{d e, j}(\mathbf{x}) \mathbf{t}_{l o c}^{d e} \mathrm{~d} A / \int_{\Gamma_{d i s}^{d e}} \bar{N}_{\Delta \dot{u}}^{d e, j}(\mathbf{x}) \mathrm{d} A, & \forall d e \in\{1, \cdots, U D E\} \text { and } j \in\{1, \cdots, 3\}
\end{array}
$$

at the strain-rate evaluation nodes, with the interpolation function for the strain-rate $N_{\dot{\varepsilon}}^{e l, i}(\mathbf{x})$ and the traction evaluation nodes with the interpolation function for velocity-jump $\bar{N}_{\Delta \dot{u}}^{d e, j}(\mathbf{x})$, respectively.

\section{Assembly}

Assembling above constraints, the primal upper bound problem Eq. (3) in discretised form reads

$$
\begin{array}{ll}
\min & W_{i n t} \\
\text { s.t. } & \mathbf{A}_{\dot{\varepsilon}}^{c o m} \mathbf{q}_{\dot{u}}=\mathbf{q}_{\dot{\varepsilon}} \\
& \mathbf{A}_{\Delta \dot{u}}^{c o m} \mathbf{q}_{\dot{u}}=\mathbf{q}_{\Delta \dot{u}} \\
& \mathbf{A}_{\Delta \dot{u}}^{l i n} \mathbf{q}_{\dot{u}}=\mathbf{0} \\
& \mathbf{q}_{\dot{u}}=\mathbf{q}_{\dot{u}_{b c}} \\
& \mathbf{R}_{\dot{\varepsilon}}^{e l} \mathbf{q}_{\dot{\varepsilon}}^{e l, i}=-\mathbf{B}_{\sigma}^{e l \top} \dot{\mathbf{e}}_{\dot{\varepsilon}}^{e l, i} \\
& \dot{\mathbf{e}}_{\dot{\varepsilon}}^{e l, i} \in \mathscr{C}^{*} \\
& \mathbf{R}_{\Delta \dot{u}}^{d e} \mathbf{q}_{\Delta \dot{u}}^{d e, j}=-\mathbf{B}_{t}^{d e} \dot{\mathbf{e}}_{\Delta \dot{u}}^{d e, j} \\
& \dot{\mathbf{e}}_{\Delta \dot{u}}^{d e, j} \in \mathscr{C}^{*}
\end{array}
$$

with $e l \in\{1, \cdots, U E\}, d e \in\{1, \cdots, U D E\}, i \in\{1, \cdots, 4\}$ and $j \in\{1, \cdots, 3\}$. In the formulation Eq. (39), $\mathbf{q}_{\Delta \dot{u}} \in \mathfrak{R}^{3 U N}$, $\mathbf{q}_{\dot{\varepsilon}} \in \Re^{24 U E}$ and $\mathbf{q}_{\Delta \dot{u}} \in \mathfrak{R}^{9 U D E}$ are the assembly of all nodal velocity vectors, nodal strain-rate vectors and nodal velocity-jump vectors over the whole structure with respect to the global coordinate basis $(x-y-z)$. Here, $U N$ is the total number of velocity evaluation nodes in the upper bound formulations. The assembled matrices are introduced as the compatibility operator in solid elements $\mathbf{A}_{\dot{\varepsilon}}^{c o m} \in \Re^{24 U E \times 3 U N}$ from Eq. (30), the compatibility operator on velocity discontinuities $\mathbf{A}_{\triangle \dot{u}}^{c o m} \in \mathfrak{R}^{9 U D E \times 3 U N}$ from Eq. (31), the operator enforcing linear velocity jumps across velocity discontinuities $\mathbf{A}_{\Delta \dot{u}}^{\text {lin }} \in \mathfrak{R}^{9 U D E \times 3 U N}$ from Eq. (32) and the prescribed kinematic boundary conditions $\mathbf{q}_{\dot{u}_{b c}} \in \mathfrak{R}^{3 U N}$ from Eq. (33).

\subsection{Dual formulations}

The dual upper bound problem Eq. (5) is based on the stress field $\sigma$ and is mathematically equivalent to the primal problem Eq. (3). As mentioned above, in the finite-element-based formulations, the numerical interpolation and integration can only be directly applied to the velocity field and the strain-rate field, thus, in the dual formulations the stress field and the traction field are interpolated using the nodal weighted-average vectors defined in Eq. (38) at evaluation nodes for the strain-rate field and the velocity field, respectively.

\section{External work rate}

The external work rate Eq. (6) in discretised form reads

$$
W_{\text {ext }}=\sum_{i=1}^{U N}\left(\mathbf{q}_{\dot{u}}^{i \top} \beta \mathbf{q}_{p_{u}}^{i}+\mathbf{q}_{\dot{u}}^{i \top} \mathbf{q}_{p_{f}}^{i}\right)
$$

where $\mathbf{q}_{u}^{i}$ is the nodal velocity field, $\mathbf{q}_{p_{u}}^{i}=\left(q_{p_{u}, x}^{i}, q_{p_{u}, y}^{i}, q_{p_{u}, z}^{i}\right)^{\top} \in \Re^{3}$ is the unknown nodal force at each velocity evaluation node determined from the unknown body force field $\mathbf{g}_{u}$ in $\Omega$ and the unknown traction field $\mathbf{t}_{u}$ on $\Gamma_{u}$, and $\mathbf{q}_{p_{f}}^{i}=\left(q_{p_{f}, x}^{i}, q_{p_{f}, y}^{i}, q_{p_{f}, z}^{i}\right)^{\top} \in \mathfrak{R}^{3}$ is the fixed nodal force determined from the fixed body force field $\mathbf{g}_{f}$ in $\Omega$ and the fixed traction field $\mathbf{t}_{f}$ on $\Gamma_{f}$. 


\section{Weak form of equilibrium}

The weak form of equilibrium refers to the equality between the total internal energy dissipation rate and the total work rate done by external loads. Considering Eqs. (36) and (40), the discretised form can be expressed as

$$
\sum_{e l=1}^{U E} \sum_{i=1}^{10} \sum_{j=1}^{4} \mathbf{q}_{\dot{u}}^{e l, i \top} \mathbf{G}_{\dot{\varepsilon}}^{e l, i, j} \hat{\mathbf{q}}_{\sigma}^{e l, j}+\sum_{d e=1}^{U D E} \sum_{i=1}^{6} \sum_{j=1}^{3} \mathbf{q}_{\dot{u}}^{d e, i \boldsymbol{\top}} \overline{\mathbf{G}}_{\Delta \dot{u}}^{d e, i, j} \hat{\mathbf{q}}_{t}^{d e, j}=\sum_{i=1}^{U N} \mathbf{q}_{\dot{u}}^{i \top}\left(\beta \mathbf{q}_{p_{u}}^{i}+\mathbf{q}_{p_{f}}^{i}\right)
$$

where it is obvious that the velocity vector at both sides can be eliminated in the assembly.

\section{Plastic yield function}

Since in the discretised formulations the stress field and the traction field are obtained as weighted-average vectors defined in Eq. (38), the second-order cone expressions of yield functions Eqs. (23) and (27) are formulated as

$$
\begin{aligned}
& \hat{\mathbf{s}}_{\sigma}^{e l, i}=\mathbf{a}_{\sigma}^{e l}+\mathbf{B}_{\sigma}^{e l} \mathbf{R}_{\sigma}^{e l} \hat{\mathbf{q}}_{\sigma}^{e l, i}, \quad \forall e l \in\{1, \cdots, U E\} \text { and } i \in\{1, \cdots, 4\} \\
& \hat{\mathbf{s}}_{\sigma}^{e l, i} \in \mathscr{C} \\
& \hat{\mathbf{s}}_{t}^{d e, j}=\mathbf{a}_{t}^{d e}+\mathbf{B}_{t}^{d e} \mathbf{R}_{t}^{d e} \hat{\mathbf{q}}_{t}^{d e, j}, \quad \forall d e \in\{1, \cdots, U D\} \text { and } j \in\{1, \cdots \\
& \hat{\mathbf{s}}_{t}^{d e, j} \in \mathscr{C}
\end{aligned}
$$

where $\mathbf{R}_{\sigma}^{e l}=\mathbf{R}_{\dot{\varepsilon}}^{e l}, \mathbf{R}_{t}^{d e}=\mathbf{R}_{\Delta \dot{u}}^{d e}$, and $\hat{\mathbf{s}}_{\sigma}^{e l, i} \in \mathfrak{R}^{8}$ and $\hat{\mathbf{s}}_{t}^{d e, j} \in \mathfrak{R}^{5}$ are the weighted-average vectors for the second-order cone expressions of the stress-based and traction-based yield functions, respectively.

\section{Assembly}

The discretised formulation of the dual upper bound problem Eq. (5) can be expressed as

$$
\begin{array}{ll}
\max & W_{e x t} \\
\text { s.t. } & \mathbf{G}_{\dot{\varepsilon}} \hat{\mathbf{q}}_{\sigma}+\overline{\mathbf{G}}_{\Delta \dot{u}} \hat{\mathbf{q}}_{t}+\mathbf{A}_{\Delta \dot{u}}^{l i n} \mathbf{r}=\beta \mathbf{q}_{p_{u}}+\mathbf{q}_{p_{f}} \\
& \hat{\mathbf{s}}_{\sigma}^{e l, i}=\mathbf{a}_{\sigma}^{e l}+\mathbf{B}_{\sigma}^{e l} \mathbf{R}_{\sigma}^{e l} \hat{\mathbf{q}}_{\sigma}^{e l, i} \\
& \hat{\mathbf{s}}_{\sigma}^{e l, i} \in \mathscr{C} \\
& \hat{\mathbf{s}}_{t}^{d e, j}=\mathbf{a}_{t}^{d e}+\mathbf{B}_{t}^{d e} \mathbf{R}_{t}^{d e} \hat{\mathbf{q}}_{t}^{d e, j} \\
& \hat{\mathbf{s}}_{t}^{d e, j} \in \mathscr{C}
\end{array}
$$

with $e l \in\{1, \cdots, U E\}$, de $\in\{1, \cdots, U D E\}, i \in\{1, \cdots, 4\}$ and $j \in\{1, \cdots, 3\}$. In the dual upper bound formulation Eq. (43), $\hat{\mathbf{q}}_{\sigma} \in \mathfrak{R}^{24 U E}$ and $\hat{\mathbf{q}}_{t} \in \mathfrak{R}^{9 U D E}$ are the assembly of the nodal weighted-average stress vectors in all solid element $\Omega^{e l}$ and the nodal weighted-average traction vectors at all discontinuities $\Gamma_{d i s}^{d e}, \mathbf{q}_{p_{u}} \in \mathfrak{R}^{3 U N}$ and $\mathbf{q}_{p_{f}} \in \mathfrak{R}^{3 U N}$ are the assembly of unknown and fixed nodal force vectors at all velocity evaluation nodes, and $\mathbf{G}_{\dot{\varepsilon}} \in \mathfrak{R}^{3 U N \times 24 U E}$ and $\overline{\mathbf{G}}_{\Delta \dot{u}} \in \Re^{3 U N \times 9 U D E}$ are the assembled matrices for integration operators from Eq. (37). The auxiliary term $\mathbf{A}_{\Delta \dot{u}}^{\operatorname{lin} T} \mathbf{r}$ in the first constraint is derived from the third constraint of the primal assembled formulation Eq. (39) due to the duality and is vanished in the internal energy expression Eq. (41) since $\mathbf{A}_{\Delta \dot{u}} \mathbf{q}_{\dot{u}}=\mathbf{0}$ where $\mathbf{r} \in \mathfrak{R}^{3 U N}$ is an auxiliary vector.

\section{Adaptive arrangement of the velocity discontinuity}

In existing finite-element-based upper bound approaches, velocity discontinuities are often introduced between all finite elements with their orientation only depending on the finite element mesh. However, in most cases significant velocity jumps occur only at very few discontinuities, in regions where very localised plastic failure modes can be identified [7, 16, 32, 33]. Thus, in many parts of a discretised body the additional degrees of freedom introduced by discontinuities are not well invested and, additionally, an in general arbitrary orientation of discontinuities prevents them to capture localised plastic flow efficiently. Subsequently, strong mesh refinements are necessary to further improve upper bound results, even if adaptive mesh refinement strategies are used. 
However, as shown by the authors in [44], if discontinuities are only introduced in regions with large plastic flow and arranged/oriented in view of potential directions of plastic flow localisation, very good upper bounds can be obtained (even for orthotropic material strength behaviour) using relatively coarse finite element meshes. In this way, only as many as useful discontinuities are implemented and, due to a sensibly arrangement, they are on average much better utilised.

Naturally, the performance of this approach relies heavily on the algorithm for the adaptive introduction and adjustment of velocity discontinuities, and its applicability to arbitrary problems. Such an algorithm will be proposed within the next sections.

\subsection{Determination of planes of plastic flow localisation}

As introduced in Section 3, plastic flow (a velocity jump) across a discontinuity will only happen when the strainrate localisation conditions in Eq. (14) are fulfilled. Thus, these conditions can also be used to find a meaningful orientation of a discontinuity. The task of a related algorithm can be formulated as: for a given strain-rate state find the critical plane on which the two tangential components and the in-plane shear component of the local strain-rate vector equal zero. In particular, for a given 3D strain-rate state $\dot{\varepsilon}=\left(\dot{\varepsilon}_{x x}, \dot{\varepsilon}_{y y}, \dot{\varepsilon}_{z z}, 2 \dot{\varepsilon}_{x y}, 2 \dot{\varepsilon}_{y z}, 2 \dot{\varepsilon}_{x z}\right)^{\top} \in \mathfrak{R}^{6}$ the critical plane $\Gamma_{\text {crit }}$ on which the local strain-rate state reads $\dot{\varepsilon}_{c r i t}=\left(\dot{\varepsilon}_{n n}, 0,0,2 \dot{\varepsilon}_{n m}, 0,2 \dot{\varepsilon}_{n p}\right)^{\top} \in \mathfrak{R}^{6}$ with respect to the local coordinate basis $(n-m-p)_{\text {crit }}$ needs to be found.

The strain-rate state can be represented by three Mohr's circles based on the three principal strain-rates $\dot{\varepsilon}_{1}, \dot{\varepsilon}_{2}$ and $\dot{\varepsilon}_{3}\left(\dot{\varepsilon}_{1} \geq \dot{\varepsilon}_{2} \geq \dot{\varepsilon}_{3}\right)$. Denoting the eigenvectors of the the strain-rate tensor as $\mathbf{v}_{1} \in \mathfrak{R}^{3}, \mathbf{v}_{2} \in \Re^{3}$ and $\mathbf{v}_{3} \in \Re^{3}$, the transformation matrix from the global coordinate basis $(x-y-z)$ into the principal coordinate basis $(1-2-3)$ reads $\mathbf{R}_{v}=\left(\mathbf{v}_{1}, \mathbf{v}_{2}, \mathbf{v}_{3}\right)^{\top} \in \Re^{3 \times 3}$.

The corresponding strain-rate invariants then read

$$
\begin{array}{ll}
\dot{I}_{1}=\dot{\varepsilon}_{1}+\dot{\varepsilon}_{2}+\dot{\varepsilon}_{3} & =\dot{\varepsilon}_{n n} \\
\dot{I}_{2}=\dot{\varepsilon}_{1} \dot{\varepsilon}_{2}+\dot{\varepsilon}_{2} \dot{\varepsilon}_{3}+\dot{\varepsilon}_{1} \dot{\varepsilon}_{3} & =-\dot{\varepsilon}_{n m}^{2}-\dot{\varepsilon}_{n p}^{2} \\
\dot{I}_{3}=\dot{\varepsilon}_{1} \dot{\varepsilon}_{2} \dot{\varepsilon}_{3} & =0
\end{array}
$$

Eqs. (44b) and (44c) are only ever met when the following conditions are satisfied

$$
\dot{\varepsilon}_{1} \geq 0 \quad \text { and } \quad \dot{\varepsilon}_{2}=0 \quad \text { and } \quad \dot{\varepsilon}_{3} \leq 0
$$

That means, if strain-rate states can be found fulfilling Eq. (45), ideal orientations for a discontinuity can be easily calculated at which plastic flow will happen very localised, as will be shown in the following.

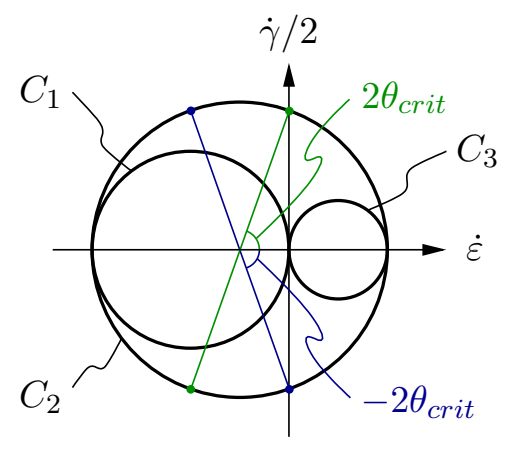

Figure 3: Mohr's circles for the given strain-rate state $\dot{\varepsilon}$ and the two critical orientations $\pm \theta_{\text {crit }}$ in the $1-3$ plane, where the tangential strain-rate components equal zero.

The Mohr's circles for the strain-rate state $\dot{\varepsilon}$ are plotted in Figure 3 as $C_{1}$ for the $2-3$ plane, $C_{2}$ for the $1-3$ plane and $C_{3}$ for the $1-2$ plane. According to Eq. (45) (since $\dot{\varepsilon}_{1} \geq 0$ ) the vertical axis must form a tangent to $C_{1}$ and $C_{3}$ and $C_{2}$ must intersect with the vertical axis since $\dot{\varepsilon}_{1} \geq 0$ and $\dot{\varepsilon}_{3} \leq 0$. Note that, in a 3D coordinate basis an arbitrary 
plane intersects with two coordinate planes if it passes through the origin $(0,0,0)$. Thus, the critical plane $\Gamma_{c r i t}$ for the strain-rate state $\dot{\varepsilon}$ can be determined from its two intersecting lines with different coordinate planes along with the tangential strain-rate component equals zero.

By means of Mohr's circles in Figure 3, since both $C_{1}$ and $C_{3}$ conform to the vertical axis, the first intersecting line runs parallel to axis 2 and its orientation is given by the normal vector $\mathbf{n}_{(123)}^{\text {line, } 1}=(0,1,0)^{\top} \in \mathfrak{R}^{3}$ with respect to the principal coordinate basis $(1-2-3)$; since $C_{2}$ intersects with the vertical axis, the second intersecting line can be determined in the $1-3$ plane. Introducing $\theta_{\text {crit }}$ as the angle between the intersecting line and axis 1 in the $1-3$ plane, based on the strain-rate transformation Eq. (8), the tangential strain-rate component along this line can be expressed as

$$
\dot{\varepsilon}_{1}\left(\cos \left(\theta_{\text {crit }}\right)\right)^{2}+\dot{\varepsilon}_{3}\left(\cos \left(\theta_{\text {crit }}\right)\right)^{2}=0
$$

resulting in

$$
\theta_{\text {crit }}= \pm \operatorname{atan}\left(\sqrt{-\dot{\varepsilon}_{1} / \dot{\varepsilon}_{3}}\right)
$$

Thus, the second intersecting line can be determined in the $1-3$ plane and exhibits two possibilities, with the normal vectors reading $\mathbf{n}_{(123)}^{\text {line,2 }}=\left(\cos \left(\theta_{\text {crit }}\right), 0, \pm \sin \left(\theta_{\text {crit }}\right)\right)^{\top} \in \mathfrak{R}^{3}$. Obviously, these two lines coincide with each other if $\dot{\varepsilon}_{1}>0, \dot{\varepsilon}_{3}=0$ then $\mathbf{n}_{(123)}^{\text {line, } 2+}=\mathbf{n}_{(123)}^{\text {line,2- }}=(0,0,1)^{\top}$ and $\dot{\varepsilon}_{1}=0, \dot{\varepsilon}_{3}<0$ then $\mathbf{n}_{(123)}^{\text {line } 2+}=\mathbf{n}_{(123)}^{\text {line } 2-}=(1,0,0)^{\top}$.

Finally, the out-of-plane normal vectors for the two possible critical planes, with respect to the global coordinate basis $(x-y-z)$, are determined as

$$
\mathbf{n}_{(x y z)}^{\text {surf, }, \pm}=\mathbf{R}_{v}^{-1} \mathbf{n}_{(123)}^{\text {surf }, \pm}=\mathbf{R}_{v}^{-1}\left(\mathbf{n}_{(123)}^{\text {line }, 1} \times \mathbf{n}_{(123)}^{\text {line }, 2 \pm}\right) \in \mathfrak{R}^{3}
$$

where $\mathbf{n}_{(123)}^{\text {surf }, \pm} \in \mathfrak{R}^{3}$ represent the normal vectors for the critical planes with respect to the principal coordinate basis $(1-2-3)$ and $\Re_{v}$ is the transformation matrix from $(x-y-z)$ to $(1-2-3)$.

\subsection{Adaptive introduction and adjustment of velocity discontinuities}

Based on the determination of critical planes, where localised plastic failure will occur, an adaptive strategy for the introduction and adjustment of velocity discontinuities $\Gamma_{d i s}$ is presented in the following. The procedure of this adaptive strategy is roughly illustrated in the flowchart Figure 4.

At first, an upper bound calculation without any velocity discontinuity using a relatively coarse mesh is performed. Based on this result, at each strain rate evaluation node (of the solid elements) the normal vectors $\mathbf{n}_{(x y z)}^{\text {surf } f,}, \mathbf{n}_{(x y z)}^{\text {surf,- }}$, indicating the two possible critical planes of plastic flow localisation, are determined. Then, the node with the highest plastic multiplier rate serves as starting point for the first introduced discontinuity. From the two possible orientations, $\mathbf{n}_{(x y z)}^{\text {surf, }}$ and $\mathbf{n}_{(x y z)}^{\text {surf,- }}$, the one is selected which points into the region with higher plastic multiplier rates. All subsequent discontinuities are introduced in a repetitive sequence, starting always at the previous end node of the last introduced discontinuity and oriented along the critical plane with the lower deviation angle to the existing discontinuity. As will be introduced in the following, the length of each discontinuity is of the same magnitude as the characteristic mesh size and defined by a straight line for problems under plane strain conditions and as an interior surface cutting through the corresponding solid element for 3D problems, respectively. In the first iteration, since a quite coarse mesh is used, the so-obtained layout for the velocity discontinuities is normally not able to capture localised failure sufficiently, and thus, adjustments through further iterations are needed, as sketched in Figure 4.

In every further iteration, the structure is discretised again, taking the introduced discontinuities into account. The improvement in the upper bound result is measured by $\Delta_{u b}(\%)=\left(\beta_{U B}^{i}-\beta_{U B}^{i-1}\right) / \beta_{U B}^{i-1} \times 100$, as the percentage reduction of the collapse load multiplier from the previous iteration $\beta_{U B}^{i-1}$ to the current iteration $\beta_{U B}^{i}$. As soon as the discontinuity layout is sufficiently arranged with respect to the appearing failure mode, only small improvements will be obtained from further iterations, and the iteration is ended as soon as $\Delta_{u b}$ is smaller than the prescribed threshold value tol.

In the following, the introduction of discontinuities is explained in more detail, separately for the plane strain case and the general 3D case.

\section{Plane strain case}

The first method is applied for problems under plane strain conditions, in which the introduction of discontinuities can be defined by lines on a 2D plane. Thereby, the $y$ axis represents the out-of-plane direction and, then, if the 


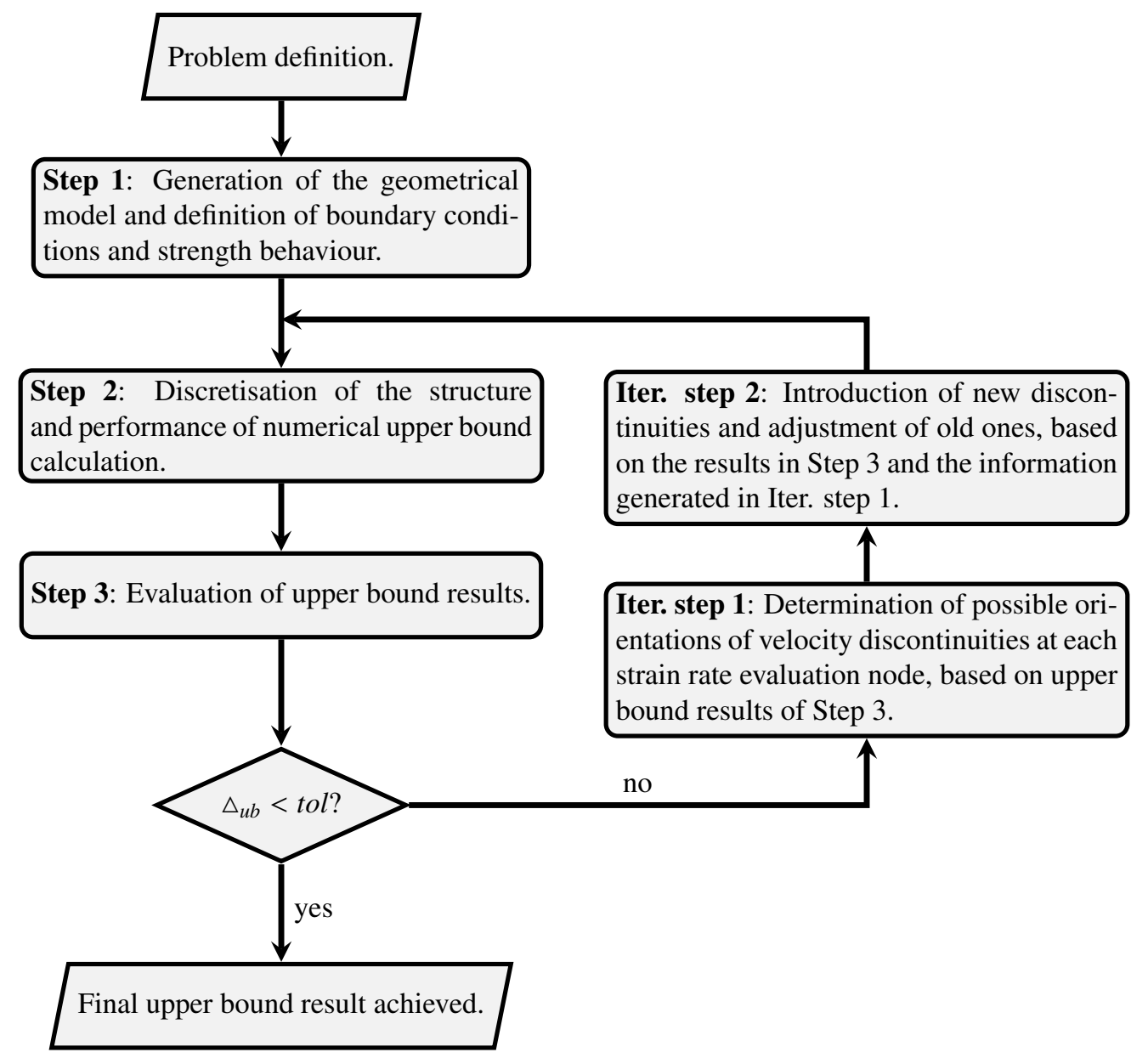

Figure 4: Flowchart introducing the adaptive strategy, where in each iteration the introduction and adjustment of velocity discontinuity (Iter. step 1-2) are based on the upper bound calculations (Step 2-3).

conditions in Eq. (45) are satisfied, one principal axis coincides with the $y$ axis and the principal strain rates $\dot{\varepsilon}_{1}, \dot{\varepsilon}_{3}$ can be found in the $x-z$ plane.

Based on an upper bound calculation, the plastic multiplier rate $\dot{\lambda}_{m a t}$ and the two normal vectors $\mathbf{n}_{(x y z)}^{\text {surf } \pm \text { according }}$ to Eq. (48), defining the critical discontinuities $\Gamma_{c r i t}$, are determined at each strain rate evaluation node. Through linear interpolation between the plastic multiplier rates and nearest neighbour interpolation between the normal vectors, the fields $\dot{\lambda}_{\text {mat }}(\hat{\mathbf{x}})$ and $\mathbf{n}_{(x y z)}^{\text {surf, }}(\hat{\mathbf{x}})$ are defined, where $\hat{\mathbf{x}}$ refers to an arbitrary node in the $x-z$ plane.

The subsequent determination of discontinuities is demonstrated in Figure 5, where the abandoned critical planes are plotted as dashed lines and the dot-dashed curves indicate contours of the plastic multiplier rate field. As illustrated in Figure 5a, the starting point of the first discontinuity is automatically chosen as the boundary node $\hat{\mathbf{x}}_{0}$ with the highest plastic multiplier rate $\dot{\lambda}_{m a t}\left(\hat{\mathbf{x}}_{0}\right)$. The end point $\hat{\mathbf{x}}_{1}$ is defined by the characteristic length of the mesh and the orientation given by the normal vectors $\mathbf{n}_{(x y z)}^{\text {surf, }}\left(\hat{\mathbf{x}}_{0}\right)$, where the orientation is selected leading to the higher plastic multiplier rate at the end point. Any further discontinuity always starts a the end node of the previous one $\hat{\mathbf{x}}_{i-1}$ and the normal vector $\mathbf{n}_{(x y z)}^{\text {surf,i }}$ is determined as the one with the smaller angle to the previous normal vector, indicated as $\hat{\theta}_{1}<\hat{\theta}_{2}$ in Figure 5a, where $i \in\left\{2, \cdots, n_{p}\right\}$ and $n_{p}$ is the total number of introduced discontinuities in this iteration. This sequence of introducing discontinuities is terminated as soon as the end node of the last line $\hat{\mathbf{x}}_{i}$ intersects with either the boundary of the 2D plane, as shown in Figure 5b, or an existing discontinuity, as shown in Figure 5c. 


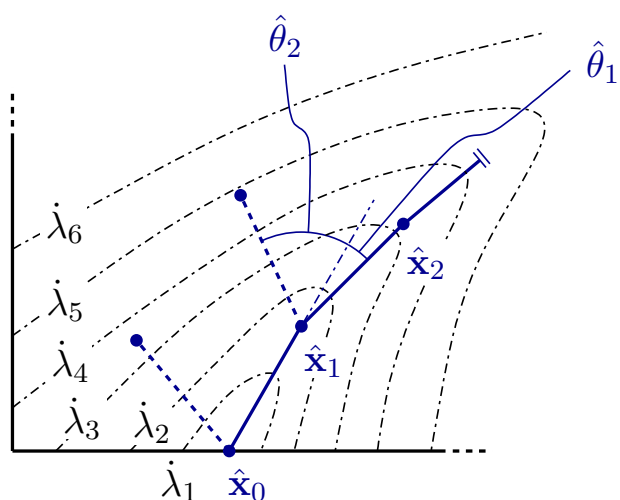

(a)

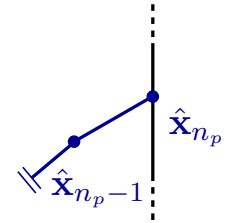

(b)

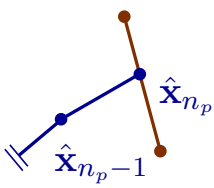

(c)

Figure 5: Illustrative example for the introduction of velocity discontinuities under plane strain conditions. (a) First and second discontinuity; (b) the last discontinuity in an iteration step in case it intersects with the boundary of the structure; (c) the last discontinuity of an iteration step in case it intersects with an existing discontinuity.

\section{General 3D case}

Instead of lines, as for the plane strain case, in 3D, discontinuities are determined and defined through surfaces. Thus, a slightly different approach is required to introduce them into a 3D body. For this reason, piecewise planar surfaces are introduced, which cut through the tetrahedral elements. To track the evolution of surfaces, the level-set method is employed $[54,55]$. Thereby, for each element, which is cut by a surface, all nodes have nonzero $\phi$-values, such that $\phi(\mathbf{x})=0$ clearly defines the partition surface. Analogously to the plane strain case, first, the element with the
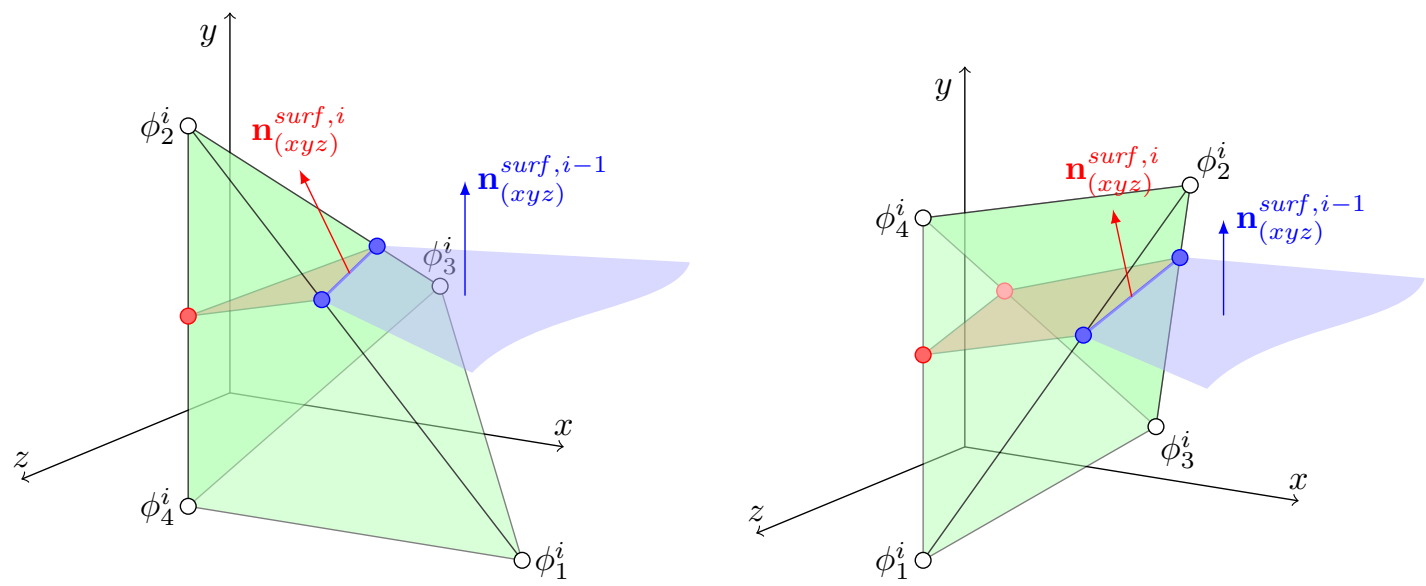

Figure 6: Two examples of an element $i$ (green) with a possible partition/discontinuity and normal vector $\mathbf{n}_{(x y z)}^{\text {surf } i}$ (red), which is adjacent to an element $i-1$ with already determined partition/discontinuity surface and normal vector $\mathbf{n}_{(x y z)}^{\text {surf } i-1}$ (blue), obtained in a previous step of the algorithm. The partition surface is tracked by using the level-set method, where the iso-zero level set $\phi(\mathbf{x})=0$ defines the surface location.

highest plastic multiplier rate $\dot{\lambda}_{\text {mat }}\left(\hat{\mathbf{x}}_{0}\right)$ on one of the boundary surfaces is chosen as the starting point of the partitioning algorithm. A first partition (in this element) with the normal vector $\mathbf{n}_{(x y z)}^{\text {surf, } 1}$ is determined from the two possibilities $\mathbf{n}_{(x y z)}^{\text {surf }, \pm}\left(\hat{\mathbf{x}}_{0}\right)$, where the one is selected leading to end nodes on the interface of the adjacent element with higher plastic multiplier rate. 
To ensure a continuous surface, the next partition is introduced in the current element following the algorithm demonstrated in Figure 6, where the blue surface indicates the determined partition surface in the adjacent element with end nodes marked in blue. Thus, three of the four $\phi$-values $\left(\phi_{1}^{i}, \phi_{2}^{i}, \phi_{3}^{i}\right)$ for the current element are already predetermined by the neighbouring element and only the last one $\left(\phi_{4}^{i}\right)$ has to be calculated. The partition with the normal vector $\mathbf{n}_{(x y z)}^{\text {surf } i}$, as the one with the smallest angle to the normal vector $\mathbf{n}_{(x y z)}^{\text {surf }, i-1}$ of the partition in the adjacent element, is chosen out of the two possible normal vectors $\mathbf{n}_{(x y z)}^{\text {surf }, \pm}\left(\hat{\mathbf{x}}_{i-1}\right)$ and the resulting fourth $\phi$-value is calculated. It can also be noticed that the resulting iso-zero level set, and, therefore, the partition surface, inside a tetrahedron is either a triangle or a quadrilateral.

This step is then repeated until no more partitioning candidates are found, which means that the piecewise planar partition has reached a boundary surface on all sides and the model can be separated into two parts, where all nodal $\phi$-values on one side of the partition have the same sign. The resulting iso-zero level set surface, which is built up of planar triangles and quadrilaterals, is then used to partition the model. Next, the partitioned model is re-meshed with a similar mesh size as the original version and the simulation is rerun with velocity discontinuities introduced between the two model parts.

\section{Numerical results}

In this section, the performance of the proposed upper bound formulations with adaptive introduction of velocity discontinuities is assessed by means of several examples. Two often used benchmark problems (under the plane strain condition) with isotropic strength behaviour are discussed and the results are compared with reference solutions, providing a basic verification of the proposed approach. Subsequently, these two examples are extended by orthotropic strength behaviour, showing that both the traction-based yield functions as well as the adaptive arrangement of velocity discontinuities also work for such cases. Finally, the full potential of the presented approach is shown by means of an example 3D problem.

It should be noted that the main goal of this section is to assess the performance of the adaptive arrangement of velocity discontinuities with respect to reliability and efficiency whereas the chosen examples themselves have no special significance. Thus, focus is laid on comparisons between upper bound results with velocity discontinuities (referred to as discontinuous upper bound results) and reference solutions obtained without any discontinuities (referred to as continuous upper bound results).

The computations were performed on a Linux desktop machine with an AMD FX(tm)-8350 processor (8 cores) and $16 \mathrm{~GB}$ of RAM. All pre- and post-processing tasks as well as the assembly of SOCP optimisation problems were carried out by self-written codes in Fortran. The mesh generation and introduction of velocity discontinuities into the discretised structure were done by the commercial software package ABAQUS controlled via self-written Python scripts. The SOCP optimisation problems themselves were solved using the commercial software MOSEK [56], which is based on the conic interior-point algorithm described in [57]. The adaptive approach introduced in Section 6 runs fully automated, realised by only a Python script for the plane strain problems and in combination with a Matlab script for general 3D problems.

\subsection{Block with thin symmetric notches}

In this benchmark problem, double-sided uniform tensile load is applied on a square block with zero-thickness symmetric notches under plane strain conditions. This example has already been extensively studied in $[58,59,19$, $20,7,31,43$ ], where very good lower as well as upper bound predictions could be obtained. An exact solution is not available for the collapse limit load multiplier $\beta$, thus, the solutions obtained based on Richardson extrapolation $[58,59]$ are considered as reference value $\beta^{*}$, with the difference between the obtained results and this reference value measured by $\operatorname{err}(\%)=\left(\beta-\beta^{*}\right) / \beta^{*} \times 100$.

For the upper bound calculation, only a quarter of the square is considered, with geometry and boundary conditions as shown in Figure 7a. $L$ is the length of each side, $a$ is the length of the notch, and $p$ refers to the uniform tensile loading. Note that, in this paper all calculations are carried out by 3D finite-element-based upper bound formulations. An example mesh with 592 tetrahedral elements is shown in Figure 7b. The plane strain condition is enforced with respect to the $x-y$ plane by applying symmetric boundary conditions on the $z-$ and $z+$ boundary surfaces. Thus, in the following, all results will be plotted only in the $x-y$ plane. 


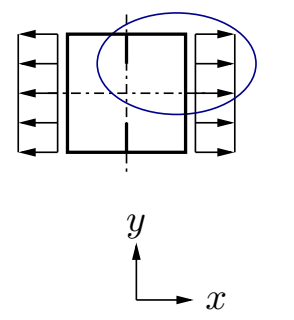

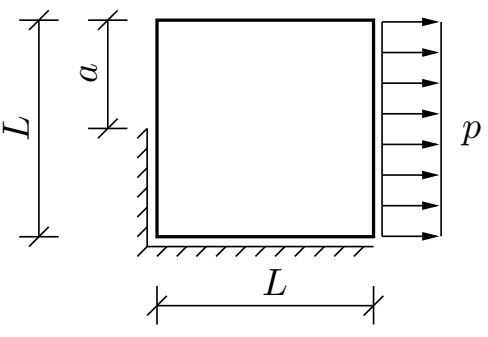

(a)

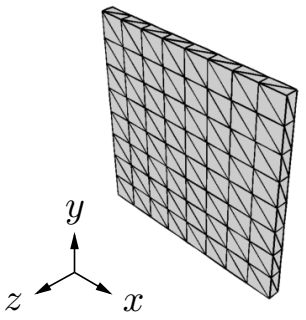

(b)

Figure 7: Example square block with symmetric notches under uniform tensile loading: (a) geometry and boundary conditions; (b) example discretisation with 592 tetrahedron elements.

Isotropic strength behaviour is defined using the von Mises yield function $\sqrt{J_{2}} \leq c$, with $J_{2}$ as the second deviatoric stress invariant and $c$ as the coefficient of cohesion. With respect to the general form of yield function in Eq. (7), the von Mises criterion is recovered by

$$
\mathbf{P}_{\sigma}^{\text {mat }}=\frac{1}{3 c^{2}}\left(\begin{array}{cccccc}
1 & -0.5 & -0.5 & 0 & 0 & 0 \\
-0.5 & 1 & -0.5 & 0 & 0 & 0 \\
-0.5 & -0.5 & 1 & 0 & 0 & 0 \\
0 & 0 & 0 & 3 & 0 & 0 \\
0 & 0 & 0 & 0 & 3 & 0 \\
0 & 0 & 0 & 0 & 0 & 3
\end{array}\right) \quad \mathbf{F}_{\sigma}^{\text {mat, },+}=\mathbf{F}_{\sigma}^{\text {mat,- }}=\mathbf{0}
$$

The traction-based yield function for velocity discontinuities is determined using the projection algorithm introduced in Section 3 and is, because of the isotropic strength behaviour, independent on the orientation of the discontinuities. The matrices according to Eq. (17c) then read

$$
\mathbf{P}_{t}^{d i s}=\frac{1}{c^{2}}\left(\begin{array}{lll}
0 & 0 & 0 \\
0 & 1 & 0 \\
0 & 0 & 1
\end{array}\right) \quad \mathbf{F}_{t}^{d i s,+}=\mathbf{F}_{t}^{d i s,-}=\mathbf{0}
$$

By means of an example with a notch length of $a=L / 2$, the performance of the adaptive introduction and arrangement of discontinuities is shown in the following. Thereby, in each iteration the improvement of the upper bound and the related failure mechanism is discussed. An upper bound value of $\lambda^{*}=1.1316$ according to [59] is chosen as reference.

\begin{tabular}{ccccccc}
\hline step & $U E$ & $U D E$ & DOFs & $\beta$ & $\operatorname{err}[\%]$ & CPU time [s] \\
\hline 1 & 395 & 0 & 2646 & 1.2532 & 10.7 & 12.8 \\
2 & 431 & 16 & 2985 & 1.1518 & 1.8 & 16.3 \\
3 & 425 & 22 & 2991 & 1.1455 & 1.2 & 18.7 \\
4 & 421 & 22 & 2970 & 1.1454 & 1.2 & 20.0 \\
\hline
\end{tabular}

Table 1: Computational performance of the proposed adaptive approach with respect to the results presented in Figure 8.

Initial step In an initial step, an upper bound calculation is performed using a relatively coarse mesh and no velocity discontinuities, as shown in Figure 8a1. The resulting failure mode (deformed configuration and plastic multiplier rate field) is plotted in Figure 8b1, where it can be observed that plastic failure is very dominant in regions close to the notch tip as well as at the corner between the loaded surface and the symmetric surface. In general, 


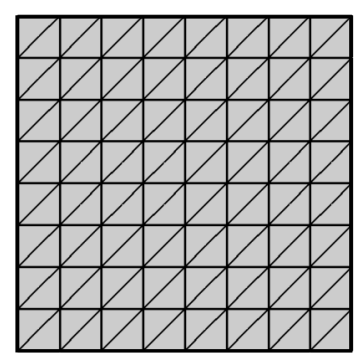

(a1) $U E=395, U D E=0$

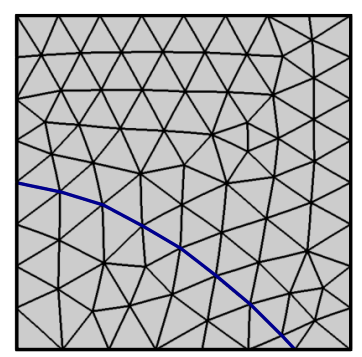

(a2) $U E=431, U D E=16$

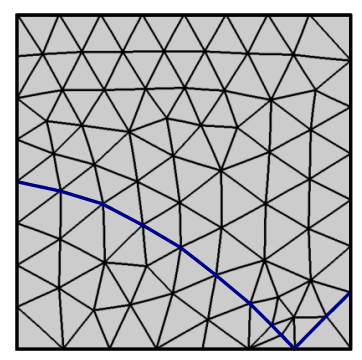

(a3) $U E=425, U D E=22$

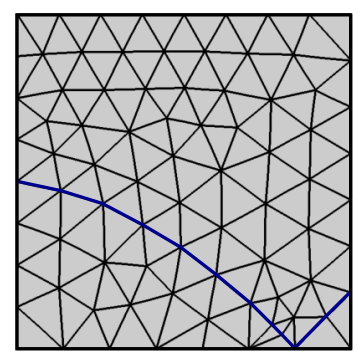

(a4) $U E=421, U D E=22$

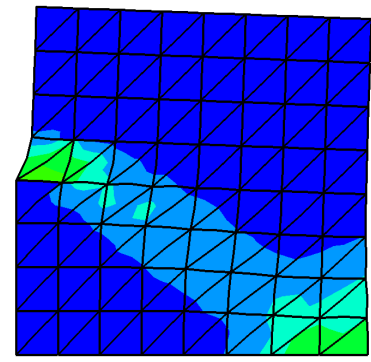

(b1) $\beta=1.2532($ err $=10.7 \%)$

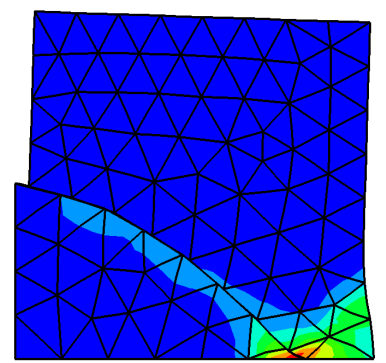

(b2) $\beta=1.1518($ err $=1.8 \%)$

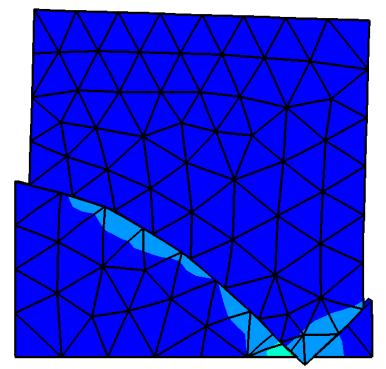

(b3) $\beta=1.1455(\mathrm{err}=1.2 \%)$

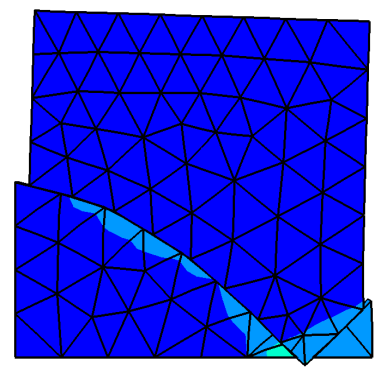

(b4) $\beta=1.1454(\mathrm{err}=1.2 \%)$

Figure 8: Example notched block under uniform tensile loading with $a=L / 2$. The adaptive introduction of velocity discontinuities through three iteration steps. (a1)-(a4) The initial mesh and the meshes for each iteration with the introduced velocity discontinuities in blue; (b1)-(b4) the related upper bound failure modes with the plastic multiplier rate $\dot{\lambda}_{\dot{\varepsilon}}^{\text {mat }}$ distribution plotted in the range $\left[0: 3.7 \times 10^{-1}\right]$. 
to the algorithm presented in Section 6, a first layout of velocity discontinuities is determined. The CPU time for this iteration step was $12.8 \mathrm{~s}$.

First iteration A first layout of velocity discontinuities is introduced into the structure as a sequence of partitions as shown in Figure 8a2. After re-meshing, the failure mode in Figure $8 \mathrm{~b} 2$ is obtained, showing significant interface shear failure across the introduced discontinuities and, simultaneously, a reduction of plastic dissipation within adjacent elements, especially within those close to the notch tip. Since the characteristic length of the introduced discontinuities is of the same size as the characteristic initial mesh size, the fineness of discretisation remains almost the same, and only a minor amount of degrees of freedom is added through the velocity discontinuities. From the initial step to this first iteration, the total number of degrees of freedom increases slightly from 2646 to 2985 but leads to an improvement of the upper bound from $10.7 \%$ error to $1.8 \%$ error. However, very high dissipation within elements can still be observed in the right bottom corner (Figure 8b2), suggesting further introduction of discontinuities. The CPU time for this iteration was $16.3 \mathrm{~s}$.

Second iteration A second iteration of the presented algorithm leads to the introduction of additional discontinuities in the right bottom corner, as illustrated in Figure 8a3. Finally, only small plastic dissipation is observed within elements and the dominant failure takes place at the introduced discontinuities. A further improvement of the collapse load multiplier from $\beta=1.1518$ to $\beta=1.1455$ is achieved, resulting in an error with respect to the reference solution of only err $=1.2 \%$. The CPU time of this iteration was $18.7 \mathrm{~s}$.

Third iteration In a third iteration the velocity discontinuities in the bottom right corner are slightly adjusted, see Figure 8a4, which doesn't significantly change the result, indicating that the velocity discontinuities are already well arranged and further iterations wouldn't improve the upper bound significantly. The CPU time for this last iteration was $20.0 \mathrm{~s}$.

This adaptive approach runs fully automated with the iteration sequence controlled by a self-written Python code, based on the strategy outlined in Figure 4. The total CPU time for this example was $74.7 \mathrm{~s}$ and the computational performance has been summarised in Table 1. Note that DOFs refers to degrees of freedoms.

For a brief study on the mesh sensitivity of the proposed approach, two different initial meshes have been used, a coarser one with 161 elements, shown in Figure 9a1, and a finer one with 4800 elements, shown in Figure 9a2, and the collapse load multipliers were obtained as $\beta=1.1737$ with err $=3.72 \%$ in a CPU time as $52.4 \mathrm{~s}$ and $\beta=1.1391$ with err $=0.66 \%$ in a CPU time of $2641.2 \mathrm{~s}$, respectively. According to Figure 9c, increasing DOFs from 1812 to 2970 (from the first data point to the second) leads to a much larger decrease on the collapse load multiplier $\beta$ than decreasing DOFs from 2970 to 25746 (from the second to the third). With respect to the upper bound failure mode, a similar pattern of adaptively introduced velocity discontinuities was determined as in the original failure mode in Figure $8 \mathrm{~b} 4$ and the refined failure mode in Figure 9b2, but the use of the coarse initial mesh led to a slightly different layout of discontinuities (see Figure 9b1) as well as a higher collapse load multiplier. The comparisons indicate that the mesh size we chose for the original illustrative example in Figure 8 has been sufficiently fine to obtain a good prediction on the localised failure mode. By using a coarser mesh, the adaptive approach is still able to provide a rough predictioin of the localised failure mode but the error increases very fast with further increase of the element size since it is too difficult for the proposed adaptive approach to identify the loacalised effect in the large elements.

Additionally, also for two other notch lengths very good results were obtained: for $a=L / 3, \beta=0.9361$ with err $=1.3 \%\left(\beta^{*}=0.9241\right)$ obtained after three iterations with a CPU time of $82.8 \mathrm{~s}$, and for $a=2 L / 3, \beta=1.4024$ with err $=1.4 \%\left(\beta^{*}=1.3833\right)$ obtained after three iterations in a CPU time of $92.4 \mathrm{~s}$. The corresponding failure modes are plotted in Figure 10, again showing almost no plastic dissipation within elements.

The obtained failure modes of the presented three examples agree very well to the ones obtained in $[59,7,31,43]$. However, in the referenced works adaptive mesh refinement is extensively used, leading to a large increase in degrees of freedom. Although the proposed results are slightly less accurate than some references (error between 1 to $2 \%$ ), due to the quite rough discretisation and few iteration steps these upper bounds could be obtained very efficient.

\subsection{Block with symmetric rectangular holes}

As second benchmark example the square block with symmetric rectangular holes under uniform load is considered. This example has also been investigated in [22, 31], where numerical limit analysis formulations with adaptive 


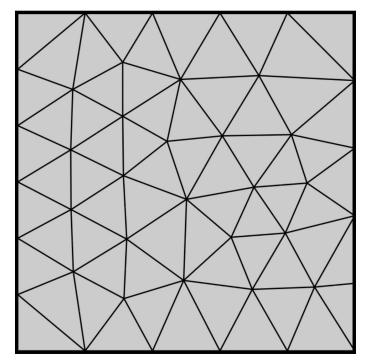

(a1) Coarse initial mesh $U E=161$

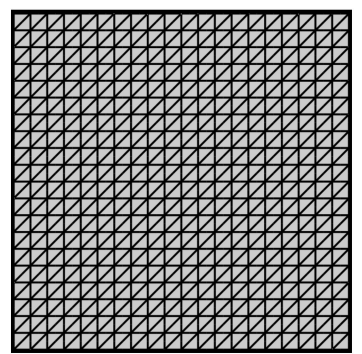

(a2) Fine initial mesh $U E=4800$

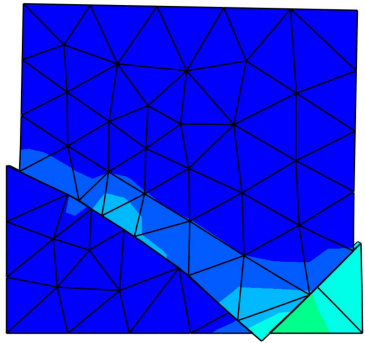

(b1) Coarse failure mode $\beta=1.1737$

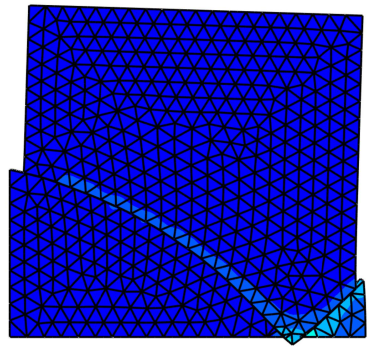

(b2) Fine failure mode $\beta=1.1391$

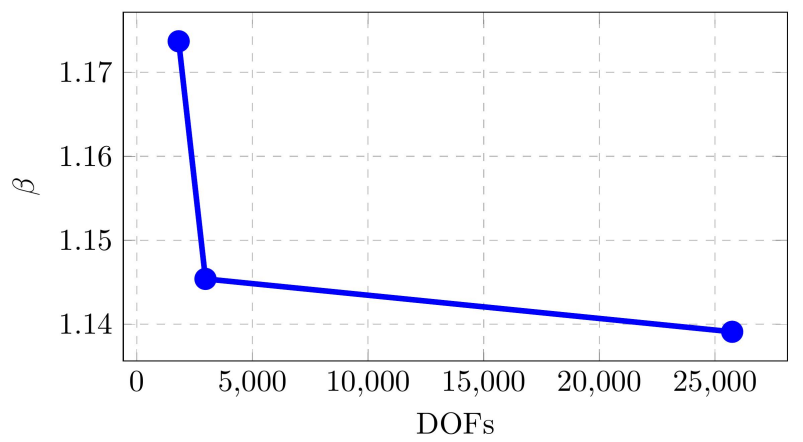

(c) The collapse load multiplier $\beta$ with respect to DOFs

Figure 9: The mesh sensitivity study by means of the example notched block under uniform tensile loading with $a=L / 2$. In failure modes (b1) and (b2), the plastic multiplier rate $\dot{\lambda}_{\dot{\varepsilon}}^{\text {mat }}$ distribution has been plotted in the range $\left[0: 1.8 \times 10^{-1}\right]$ and $\left[0: 4.8 \times 10^{-1}\right]$, respectively.

mesh refinement led to reliable and very good results. However, in these references only purely cohesive material behaviour under uniform tensile load has been considered. A more interesting problem is the failure behaviour of the block under uniform compressive load with cohesive frictional strength behaviour, as stated in [33].

The considered geometry and boundary conditions are plotted in Figure 11a, and an exemplary 3D mesh of the model with 618 tetrahedral elements is shown in Figure 11b, where symmetric boundary conditions are prescribed on the $z-$ and $z+$ boundary surfaces. A cohesive frictional strength behaviour is defined through the Drucker-Prager yield function $\sqrt{J_{2}}=A-B I_{1}$, with $I_{1}$ as the first stress invariant. Since the Drucker-Prager yield function and the Mohr-Coulomb yield function are equivalent to each other under plane strain conditions, the strength parameters are determined for two different friction angles $\phi$, reading

$$
\begin{array}{lll}
A=c & B=0 & \text { for } \quad \phi=0^{\circ} \\
A=0.8321 c & B=0.1601 & \text { for } \quad \phi=30^{\circ}
\end{array}
$$




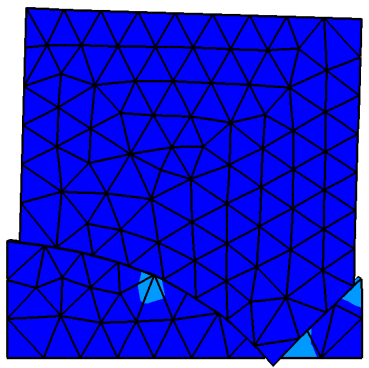

(a) $a=L / 3$

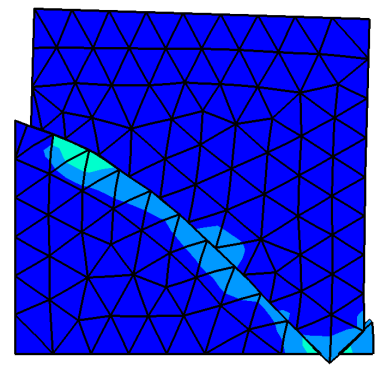

(b) $a=2 L / 3$

Figure 10: Example notched block under uniform tensile loading. (a) $a=L / 3$ with $U E=581, U D E=22$ and $\dot{\lambda}_{\dot{\varepsilon}}^{\text {mat }}$ in the range $\left[0: 4.5 \times 10^{-3}\right]$; (b) $a=2 L / 3$ with $U E=578, U D E=24$ and $\dot{\lambda}_{\dot{\varepsilon}}^{\text {mat }}$ in the range $\left[0: 6.2 \times 10^{-3}\right]$.

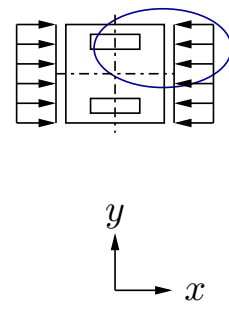

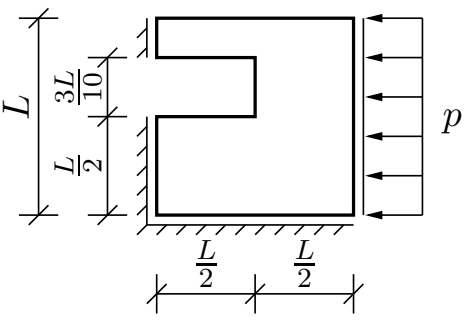

(a)

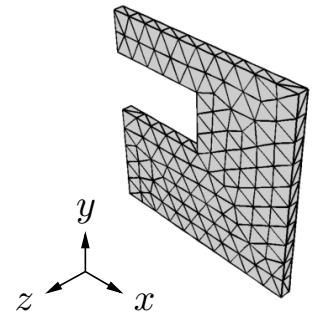

(b)

Figure 11: Example square block with symmetric rectangular holes under uniform compressive loading. (a) Geometry and boundary conditions; (b) Exemplary mesh with 618 tetrahedron elements.

For $\phi=0^{\circ}$ the Drucker-Prager yield function reduces to the von Mises yield function and the strength parameters according to Eq. (49) and Eq. (7), respectively, are used. For $\phi=30^{\circ}$ the matrices defining the yield functions become

$$
\mathbf{P}_{\sigma}^{\text {mat }}=\frac{1}{3 A^{2}}\left(\begin{array}{cccccc}
1 & -0.5 & -0.5 & 0 & 0 & 0 \\
-0.5 & 1 & -0.5 & 0 & 0 & 0 \\
-0.5 & -0.5 & 1 & 0 & 0 & 0 \\
0 & 0 & 0 & 3 & 0 & 0 \\
0 & 0 & 0 & 0 & 3 & 0 \\
0 & 0 & 0 & 0 & 0 & 3
\end{array}\right) \quad \mathbf{F}_{\sigma}^{\text {mat, },+}=\mathbf{0} \quad \mathbf{F}_{\sigma}^{\text {mat },-}=\frac{B}{A}\left(\begin{array}{l}
1 \\
1 \\
1 \\
0 \\
0 \\
0
\end{array}\right)
$$

for the stress-based yield function according to Eq. (7) and

$$
\mathbf{P}_{t}^{d i s}=\frac{\left(1-12 B^{2}\right)}{A^{2}}\left(\begin{array}{lll}
0 & 0 & 0 \\
0 & 1 & 0 \\
0 & 0 & 1
\end{array}\right) \quad \mathbf{F}_{t}^{d i s,+}=\mathbf{0} \quad \mathbf{F}_{t}^{d i s,-}=\frac{B}{A}\left(\begin{array}{l}
1 \\
0 \\
0
\end{array}\right)
$$

for the traction-based yield function according to Eq. (17c).

For this example, in the following only the upper bound results of the initial step and of the last iteration are discussed. For purely cohesive strength behaviour, $\phi=0^{\circ}$, an initial calculation is performed using a mesh with only 192 elements, as plotted in Figure 12a1. The corresponding failure mode is shown in Figure 12b1, where two separated regions with high plastic dissipation can be identified. Based on that initial result, after three iterations and the introduction of 10 discontinuities a final layout of velocity discontinuities was obtained, shown in Figure 12a2. The 


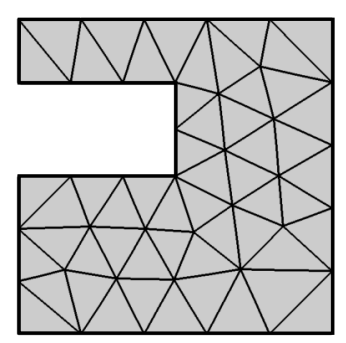

(a1) $U E=192, U D E=0$

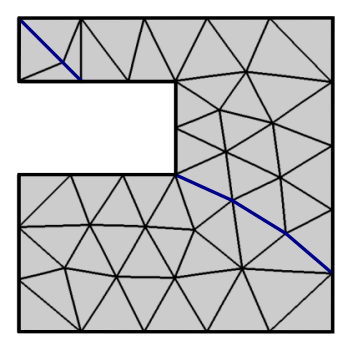

(a2) $U E=204, U D E=10$

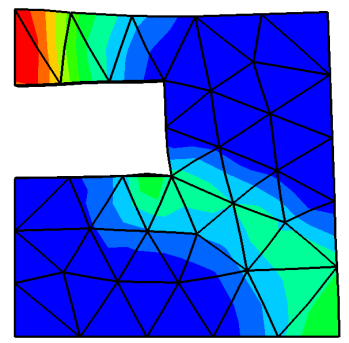

(b1) $\beta=1.4265$

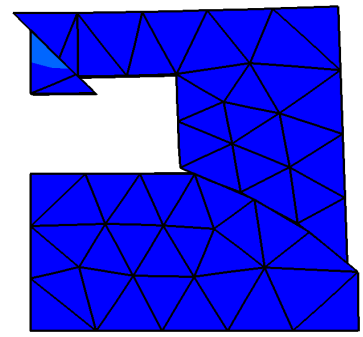

(b2) $\beta=1.3432$

Figure 12: Example block with symmetric rectangular holes under uniform compressive loading, considering purely cohesive strength behaviour. (a1) Discretised structure for the initial calculation; (a2) discretised structure with velocity discontinuities (blue), determined through three iterations; (b1) and (b2) related failure modes with $\dot{\lambda}_{\dot{\varepsilon}}^{\text {mat }}$ plotted in the range $\left[0: 1.05 \times 10^{-2}\right]$.

\begin{tabular}{cccccc}
\hline step & $U E$ & $U D E$ & DOFs & $\beta$ & CPU time [s] \\
\hline 1 & 192 & 0 & 1344 & 1.4265 & 9.9 \\
2 & 191 & 4 & 1395 & 1.4304 & 13.2 \\
3 & 204 & 10 & 1509 & 1.3432 & 15.4 \\
4 & 206 & 16 & 1602 & 1.3492 & 14.5 \\
\hline
\end{tabular}

Table 2: Computational performance of the proposed adaptive approach with respect to the results presented in Figure 12.

total number of degrees of freedom increased from 1344 to 1509 . The final failure mode, illustrated in Figure 12b2, again shows that plastic dissipation can be captured very localised by the introduced discontinuities. During the three iterations, the upper bound collapse multiplier $\beta$ reduced from 1.4265 to 1.3432 and the total CPU time was $53.0 \mathrm{~s}$. The computational performance has been summarised in Table 2 .

\begin{tabular}{cccccc}
\hline step & $U E$ & $U D E$ & DOFs & $\beta$ & CPU time [s] \\
\hline 1 & 192 & 0 & 1344 & 2.2214 & 10.2 \\
2 & 180 & 4 & 1350 & 2.2237 & 11.5 \\
3 & 180 & 4 & 1350 & 2.2218 & 12.8 \\
4 & 192 & 10 & 1464 & 2.0764 & 12.9 \\
4 & 204 & 12 & 1557 & 2.0754 & 16.7 \\
\hline
\end{tabular}

Table 3: Computational performance of the proposed adaptive approach with respect to the results presented in Figure 13.

For cohesive-frictional strength behaviour, $\phi=30^{\circ}$, the discretised structures and the corresponding results are 


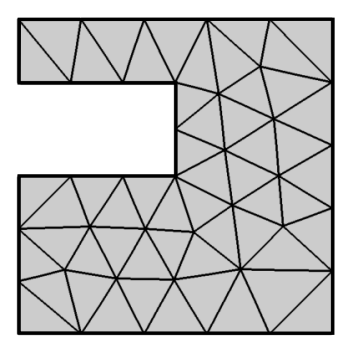

(a1) $U E=192, U D E=0$

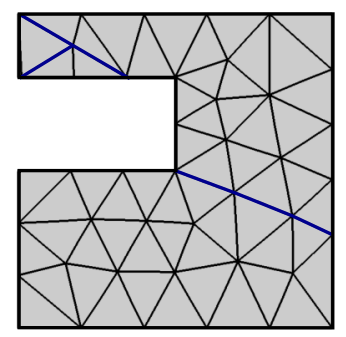

(a2) $U E=204, U D E=12$

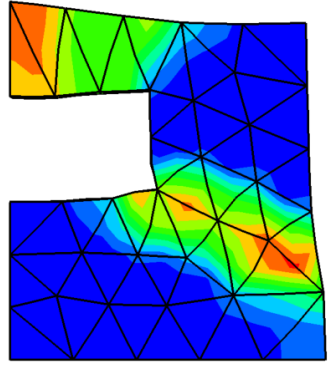

(b1) $\beta=2.2214$

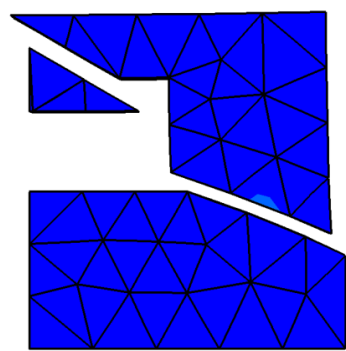

(b2) $\beta=2.0754$

Figure 13: Example block with symmetric rectangular holes under uniform compressive loading, considering cohesive-frictional strength behaviour, $\phi=30^{\circ}$. (a1) Discretised structure for the initial calculation; (a2) discretised structure with velocity discontinuities (blue), determined through four iterations; (b1) and (b2) related failure modes with $\dot{\lambda}_{\dot{\varepsilon}}^{\text {mat }}$ plotted in the range $\left[0: 1.1 \times 10^{-2}\right]$.

shown in Figure 13. Again, through the introduction of only a few velocity discontinuities the very localised failure could be captured appropriately. Since cohesive-frictional strength behaviour is considered, not only shear failure but also normal velocity jumps across discontinuities can be observed. In this way, the upper bound collapse multiplier $\beta$ was improved from 2.2214 to 2.0754 , at a total CPU time of $69.3 \mathrm{~s}$. The computational performance has been summarised in Table 3 .

\begin{tabular}{ccccc}
\hline Authors & LB/UB & UE & $\beta\left(\phi=0^{\circ}\right)$ & $\beta\left(\phi=30^{\circ}\right)$ \\
\hline \multirow{2}{*}{ Ciria and Peraire[7] } & LB & 4788 & 1.3219 & - \\
& UB & 4788 & 1.3342 & - \\
\hline \multirow{2}{*}{ Makrodimopoulos and Martin [33] } & LB & 34000 & 1.3221 & 2.0605 \\
& UB & 4788 & 1.3290 & 2.0840 \\
& UB & 34000 & 1.3260 & 2.0705 \\
\hline Nguyen-Xuan and Liu [31] & UB & 4208 & 1.3247 & - \\
\hline present & UB & 204 & 1.3432 & 2.0754 \\
\hline
\end{tabular}

Table 4: Comparison of obtained (present) collapse limit load multiplier $\beta$ with reference solutions, including the number of elements (UE).

Since no exact solution is available for this problem, the collapse load multiplier $\beta$ obtained from our calculations are compared with those available in references listed in Table 4 . Note that for this example, when purely cohesive strength behaviour is considered, $\phi=0^{\circ}$, the collapse limit load multipliers $\beta$ obtained from uniform tensile load $([7,31])$ and compressive load ([33] and present work) are equivalent to each other. Table 4 shows that the present upper bound approach is able to provide quite good strength predictions by using much less elements compared to the reference solutions, even though 3D tetrahedral elements are used in our calculation versus $2 \mathrm{D}$ triangular elements 
used in references.

\subsection{Examples with orthotropic strength behaviours}

In the following, the performance of the presented approach including orthotropic strength behaviour is assessed by means of the two examples above. Therefore, a Tsai-Wu yield function is assigned to the material, defined by inserting

$$
\mathbf{P}_{\sigma}^{m a t}=\left(\begin{array}{cccccc}
P_{11} & P_{12} & P_{13} & 0 & 0 & 0 \\
P_{12} & P_{22} & P_{23} & 0 & 0 & 0 \\
P_{13} & P_{23} & P_{33} & 0 & 0 & 0 \\
0 & 0 & 0 & P_{44} & 0 & 0 \\
0 & 0 & 0 & 0 & P_{55} & 0 \\
0 & 0 & 0 & 0 & 0 & P_{66}
\end{array}\right) \quad \mathbf{F}_{\sigma}^{m a t,+}=\mathbf{F}_{\sigma}^{m a t,-}=\left(\begin{array}{c}
F_{1} \\
F_{2} \\
F_{3} \\
0 \\
0 \\
0
\end{array}\right)
$$

into the general stress-based yield function in Eq. 7.

The strength parameters are chosen in a way that they represent the behaviour of Norway spruce clear wood, a classical orthotropic material, as introduced in [60], giving

$$
\mathbf{P}_{\sigma}^{m a t}=\left(\begin{array}{cccccc}
0.0002 & 0 & 0 & 0 & 0 & 0 \\
0 & 0.0659 & 0 & 0 & 0 & 0 \\
0 & 0 & 0.0659 & 0 & 0 & 0 \\
0 & 0 & 0 & 0.0473 & 0 & 0 \\
0 & 0 & 0 & 0 & 0.1189 & 0 \\
0 & 0 & 0 & 0 & 0 & 0.0473
\end{array}\right) \quad \mathbf{F}_{\sigma}^{\text {mat, }}=\mathbf{F}_{\sigma}^{\text {mat },-}=\left(\begin{array}{c}
-0.0066 \\
0.0856 \\
0.0856 \\
0 \\
0 \\
0
\end{array}\right)
$$

with respect to the material local coordinate basis $(L-R-T)$. The traction-based yield function for each velocity discontinuity is determined according to the projection algorithm introduced in Section 3.

\begin{tabular}{cccccc}
\hline step & $U E$ & $U D E$ & DOFs & $\beta$ & CPU time [s] \\
\hline 1 & 216 & 0 & 1521 & 2.3705 & 9.6 \\
2 & 264 & 8 & 1857 & 2.0785 & 19.8 \\
3 & 264 & 6 & 1836 & 2.0785 & 12.5 \\
\hline
\end{tabular}

Table 5: Computational performance of the proposed adaptive approach with respect to the results presented in Figure 14.

Firstly, the example illustrated in Figure 7a with a notch length of $a=L / 2$ is investigated. The arbitrary chosen longitudinal direction $L$ is defined in Figure 14a1, also showing the mesh for the initial calculation. The corresponding failure mode is given in Figure 14b1, showing a concentration of plastic dissipation rate around the notch tip, but no distinct failure surface / slip line can be identified. In the course of two iterations 8 velocity discontinuities were introduced (see Figure 14a2), almost parallel to the longitudinal direction $L$, increasing the total number of degrees of freedom from 1521 to 1857 and the number of elements from 216 to 264 . The final failure mechanism is shown in Figure 14b2, where almost all plastic failure occurs across discontinuities. Through the whole iteration process, the upper bound collapse load multiplier $\beta$ decreases from 2.3705 to 2.0785 , within a CPU time of $47.5 \mathrm{~s}$. The computational performance has been summarised in Table 5.

Since neither an exact solution nor a reference value is available for this example, the obtained upper bounds are compared to a solution without velocity discontinuities but with a very fine mesh consisting of 55571 tetrahedral elements, shown in Figure 16a. Additionally, the 3D lower bound formulations proposed by the authors in [50] was applied to enclose the exact collapse load multiplier $\beta^{*}$, giving

$$
1.7855<\beta^{*}<2.0878
$$




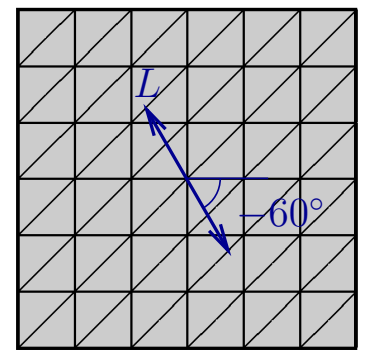

(a1) $U E=216, U D E=0$

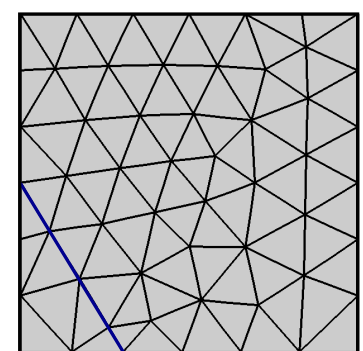

(a2) $U E=264, U D E=8$

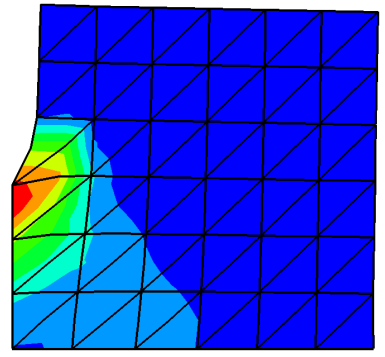

(b1) $\beta=2.3705$

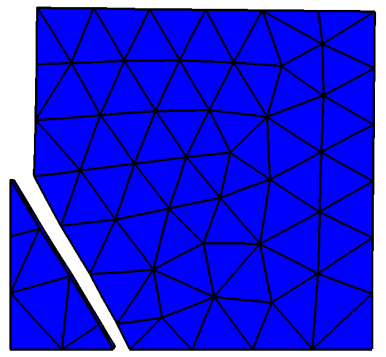

(b2) $\beta=2.0785$

Figure 14: Example block with symmetric notches $(a=L / 2)$ under uniform tensile loading considering orthotropic strength behaviour. (a1) Discretised structure for the initial calculation and the prescribed longitudinal direction $L$ (blue arrow); (a2) discretised structure with velocity discontinuities (blue), determined through two iterations; (b1) and (b2) related failure modes with $\dot{\lambda}_{\dot{\varepsilon}}^{\text {mat }}$ plotted in the range [0: $\left.4 \times 10^{-2}\right]$.

The total CUP time for the upper bound as well as lower bound calculation with the fine mesh were 10.6 min and 18.6 min, respectively. The upper bound obtained with velocity discontinuities, $\beta=2.0785$, can thus be considered as reliable, since it is located within the bound gap given in Eq. (56). It is even slightly better than the upper bound obtained using the very fine mesh.

\begin{tabular}{cccccc}
\hline step & $U E$ & $U D E$ & DOFs & $\beta$ & CPU time [s] \\
\hline 1 & 192 & 0 & 1344 & 5.9887 & 12.0 \\
2 & 175 & 4 & 1329 & 5.9812 & 24.4 \\
3 & 190 & 10 & 1449 & 5.7743 & 12.5 \\
4 & 175 & 10 & 1383 & 5.7742 & 17.0 \\
\hline
\end{tabular}

Table 6: Computational performance of the proposed adaptive approach with respect to the results presented in Figure 15.

Secondly, the orthotropic strength behaviour is applied to the example defined in Figure 11a, with the longitudinal direction $L$ defined in Figure 15a1. The discontinuity layout shown in Figure 15a2 was obtained after three iterations, the introduction of 10 discontinuities, and an increase of the total number of degrees of freedom from 1344 to 1383. The collapse load multiplier decreased from 5.9887 to 5.7742 within a CPU time of $71.8 \mathrm{~s}$. Note that, in this example, the number of elements even decreases from 192 to 175, being an output of the proposed algorithm without any manual influence. The computational performance has been summarised in Table 6 .

Again, the result is compared to upper and lower bounds obtained with an approach without velocity discontinuities but a very fine mesh with 56894 tetrahedral elements, as displayed in Figure 16b, giving

$$
5.6681<\beta^{*}<5.7981
$$

in a CPU time of $12.5 \mathrm{~min}$ and $24.0 \mathrm{~min}$, respectively. Also for this example, the upper bound obtained with a discon- 


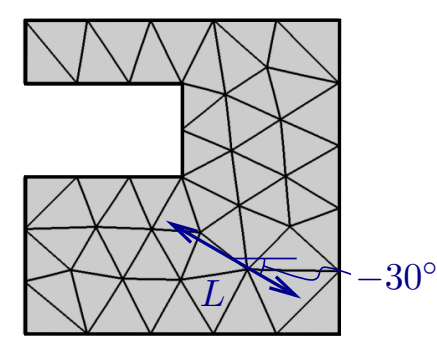

(a1) $U E=192, U D E=0$

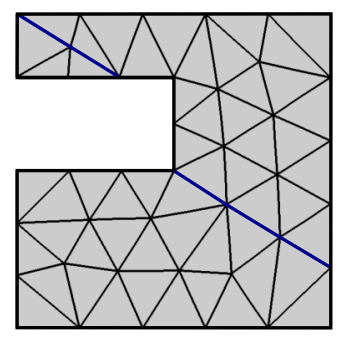

(a2) $U E=175, U D E=10$

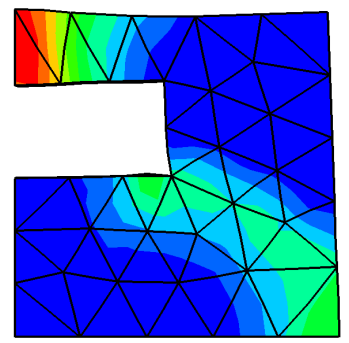

(b1) $\beta=5.9887$

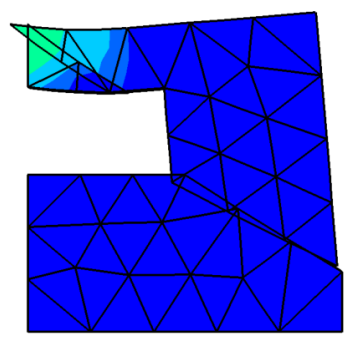

(b2) $\beta=5.7742$

Figure 15: Example block with symmetric rectangular holes under uniform compressive loading considering orthotropic strength behaviour. (a1) Discretised structure for the initial calculation and the prescribed longitudinal direction $L$ (blue arrow); (a2) discretised structure with velocity discontinuities (blue), determined through three iterations; (b1) and (b2) related failure modes with $\dot{\lambda}_{\dot{\varepsilon}}^{\text {mat }}$ plotted in the range $\left[0: 6 \times 10^{-2}\right]$.

tinuous velocity field and a very rough mesh represents a reliable solution and the best upper bound. Comparing the failure modes of these two approaches, Figure 16b versus Figure 15b2, it becomes obvious that velocity discontinuities can only unfold their full potential if there is really localised failure, as in the lower right part of this example. In case of distributed plastic collapse, as in the upper left region of this example, discontinuities are, clearly, not able to take over plastic dissipation. Thus, there is still plastic dissipation within solid elements after the last iteration, as can be seen in Figure 15b2. Nevertheless, both examples show that the presented approach works also very reliable and

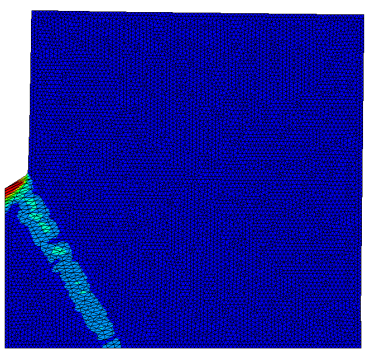

(a) $U E=55571, \beta=2.0878$

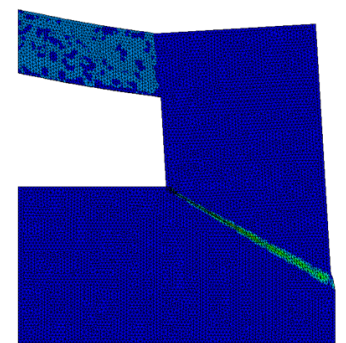

(b) $U E=56894, \beta=5.7981$

Figure 16: Upper bound failure modes for the two examples with orthotropic strength behaviour using a fine mesh and no velocity discontinuities. (a) block with symmetric notches under uniform tensile loading with $\dot{\lambda}_{\dot{\varepsilon}}^{\text {mat }}$ plotted in the range [0 : 0.0015]; (b) Block with symmetric rectangular holes under uniform compressive loading with $\dot{\lambda}_{\dot{\varepsilon}}^{\text {mat }}$ plotted in the range $\left[0: 1 \times 10^{-3}\right]$.

efficient for problems with orthotropic strength behaviour. 


\subsection{D cylinder with central circular notch}

As a first 3D example a cylinder with central circular notch is considered, applying the algorithm presented for the general 3D case instead of the plane strain case. Thus, the introduced discontinuities not necessarily form a plane surface.
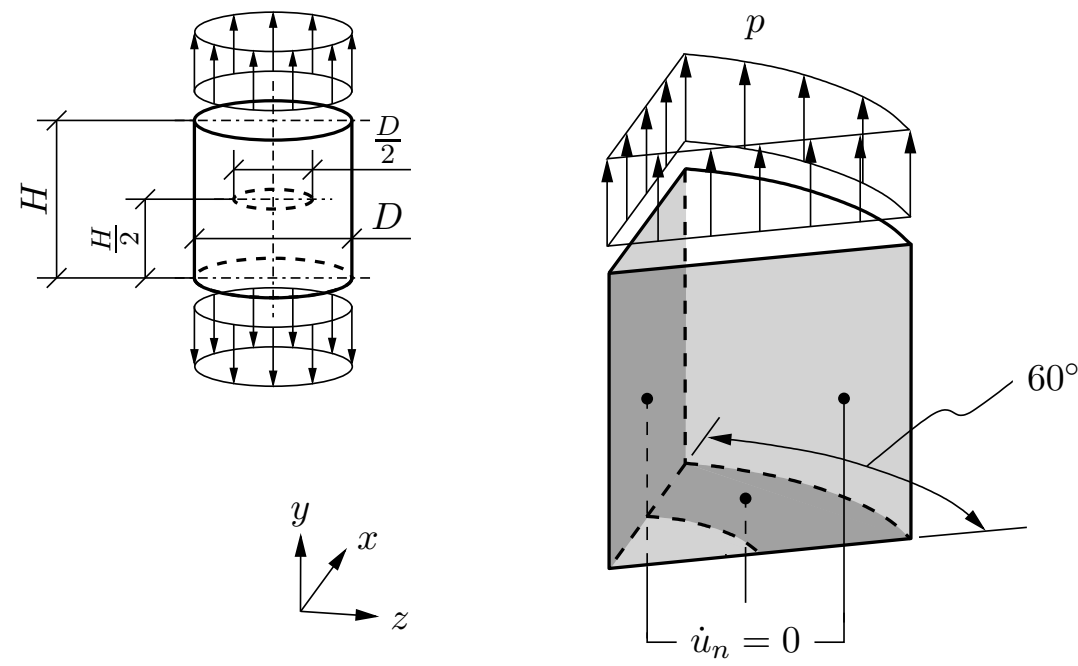

Figure 17: Example cylinder $(H=D)$ with central circular notch under uniform tensile loading $p$, and $60^{\circ}$ slice used for upper bound calculations.

The geometry and boundary conditions are defined in Figure 17. For the upper bound calculation, a $60^{\circ}$ slice of the upper-half of the cylinder is considered. Cohesive-frictional isotropic strength behaviour is assigned using the Drucker-Prager yield function according to Eq. (52), with the parameters $A=0.9219 c$ and $B=0.1118$. Again, according to the projection algorithm in Section 3, the traction-based yield function for the strength behaviour across velocity discontinuities is determined according to Eq. (53), which is identical for all orientations due to the isotropic strength behaviour.

\begin{tabular}{cccccc}
\hline step & $U E$ & $U D E$ & DOFs & $\beta$ & CPU time [s] \\
\hline 1 & 923 & 0 & 4707 & 1.1184 & 41.0 \\
2 & 995 & 56 & 5190 & 1.0565 & 90.0 \\
\hline
\end{tabular}

Table 7: Computational performance of the proposed adaptive approach with respect to the results presented in Figure 18.

Similar to the previous examples, an initial upper bound calculation is performed using a coarse mesh with 923 tetrahedral elements, as shown in Figure 18a1. The corresponding failure mode is displayed in Figure 18b1, leading to an upper bound of the collapse load multiplier of $\beta=1.1184$. A very high plastic dissipation rate can be observed around the notch tip, but a distinct localised failure region can hardly be identified.

Based on this initial result, the algorithm proposed in Section 6 for the general 3D case was applied, making use of the level-set method as illustrated in Figure 6 to define the geometry of discontinuities to be introduced. By applying this algorithm, a sequence of continuous discontinuities, plotted in Figure 19a, are determined, cutting through the tetrahedral elements. Since the so-obtained discontinuities are either triangular or quadrilateral plane surfaces in various sizes, it is not ideal to use the resulting surface directly for re-meshing the whole structure. For this reason, the Face from mesh feature in ABAQUS/CAE is used for smoothing, resulting in the surface shown in Figure 19b. Since this smoothed surface can be discretised using triangular elements of uniform size, it is guaranteed that the new mesh created for the next iteration is balanced and of similar mesh size as the initial mesh. Performing only one iteration, 56 discontinuities were introduced and the structure was re-meshed with 995 tetrahedral elements, as shown in Figure 18a2. The total number of degrees of freedom is 5190. 


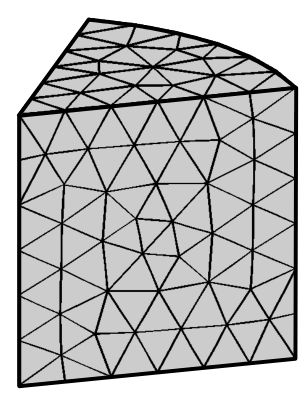

(a1) $U E=923, U D E=0$

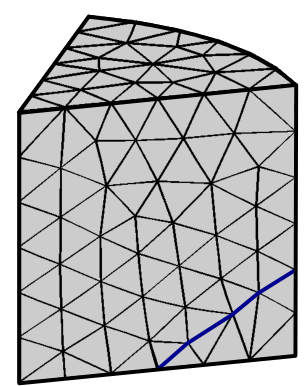

(a2) $U E=995, U D E=56$

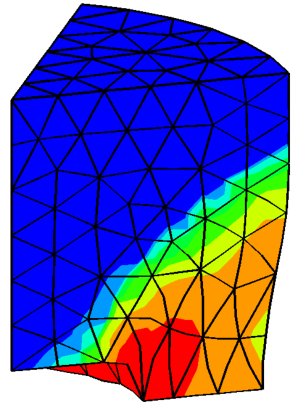

(b1) $\beta=1.1184$

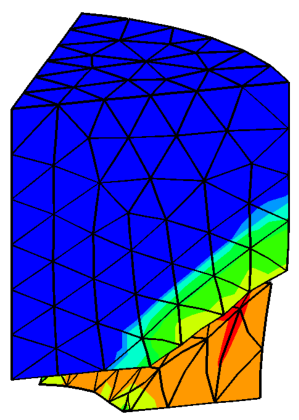

(b2) $\beta=1.0565$

Figure 18: Example cylinder with central circular notch under uniform tensile loading, considering cohesive-frictional isotropic strength behaviour. (a1) Discretised structure used in the initial calculation; (a2) discretised structure with velocity discontinuities (blue); (b1) and (b2) related failure modes with $\dot{\dot{\varepsilon}}_{\dot{\varepsilon}}^{\text {mat }}$ plotted in the range $\left[1 \times 10^{-6}: 3.3 \times 10^{-3}\right]$.

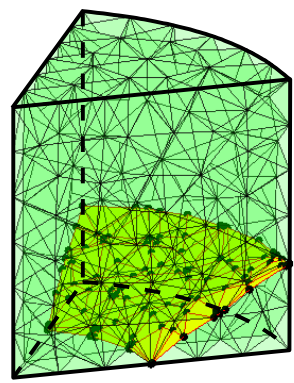

(a)

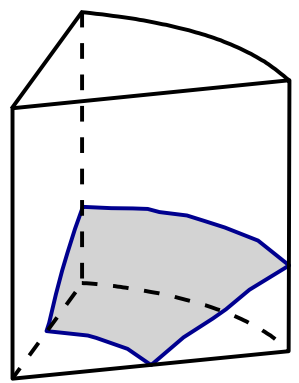

(b)

Figure 19: Example cylinder with central circular notch under uniform tensile loading. (a) Continuous partitions cutting through tetrahedral elements defined by the level-set method introduced in Figure 6; (b) resulting smooth surface determined from the piecewise plane partition surfaces using the Face from mesh feature in ABAQUS/CAE.

Finally, a distinct plastic flow across the introduced velocity discontinuities is obtained (Figure 18b2), and the plastic dissipation rate within the solid elements around the notch tip is reduced significantly. A continuous upper bound calculation with 88798 tetrahedral elements (379593 degrees of freedom) was performed to obtain a reference 


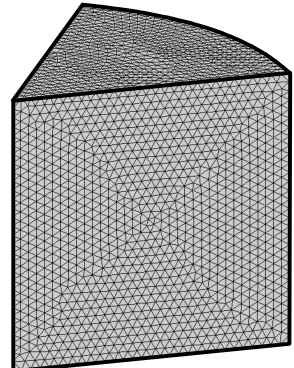

(a)

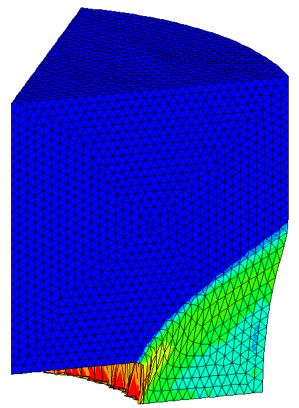

(b)

Figure 20: Example cylinder with central circular notch under uniform tensile loading. (a) Fine mesh with 88798 tetrahedral elements and without velocity discontinuities, and (b) the corresponding continuous upper bound failure mode with the collapse load multiplier $\beta=1.0547$.

solution. The mesh as well as the corresponding failure mode is shown in Figure 20a and Figure 20b, respectively. The failure modes agree very well and the collapse load multiplier of the discontinuous upper bound approach, $\beta=1.0565$, is only slightly larger than the one of the continuous approach, $\beta=1.0547$. However, the total CPU time of the continuous approach was $131.7 \mathrm{~min}$, whereas the discontinuous approach only took $131.0 \mathrm{~s}$. $41.0 \mathrm{~s}$ for the initial upper bound calculation, $44.1 \mathrm{~s}$ for the introduction of velocity discontinuities, and $45.9 \mathrm{~s}$ for the discontinuous upper bound calculation. The computational performance has been summarised in Table 7.

Note that, in the discontinuous failure mode in Figure 18b2, the high plastic dissipation rate (red and orange regions) within tetrahedral elements beneath velocity discontinuities indicates a mixed mode of shear and tensile failure which is rather dispersed, as also observed in the continuous failure mode in Figure 20b. It is also worth mentioning that, in this paper, only one iteration is used for the introduction of velocity discontinuities for general 3D problems and no further adjustment is included. This is because the introduction of velocity discontinuities is based on the framework of the level-set method, and the adjustment of an introduced discontinuity as well as the intersection between a new discontinuity with an existing one has not yet been implemented and is considered as a difficulty to be overcome in future work.

\section{5. $3 D$ cylinder with half-circular notch}

As final example the previous one is slightly modified by introducing a half-circular notch into the 3D cylinder, which results in a 3D failure mechanism compared to the axisymmetric one in the last example.

The geometry and boundary conditions are defined in Figure 21 and for the upper bound calculation the upper front quarter of the cylinder is considered. Again, cohesive-frictional isotropic strength behaviour is assigned using the Drucker-Prager yield function according to Eq. (52), with the parameters $A=0.9219 c$ and $B=0.1118$, and according to the projection algorithm in Section 3, the traction-based yield function for the strength behaviour across velocity discontinuities is determined according to Eq. (53), which is identical for all orientations due to the isotropic strength behaviour.

\begin{tabular}{cccccc}
\hline step & $U E$ & $U D E$ & DOFs & $\beta$ & CPU time $[\mathrm{s}]$ \\
\hline 1 & 796 & 0 & 4065 & 0.7896 & 26.5 \\
2 & 1386 & 49 & 6789 & 0.7122 & 34.0 \\
ref & 36020 & 0 & 156468 & 0.7241 & 687.9 \\
\hline
\end{tabular}

Table 8: Computational performance of the proposed adaptive approach with respect to the results presented in Figures 22 and 24.

The initial upper bound calculation with a coarse mesh (796 tetrahedral elements) is shown in Figure 22a1. As expected this leads to a rather dispersed failure mechanism (see Figure 22b1) and an upper bound of the collapse 

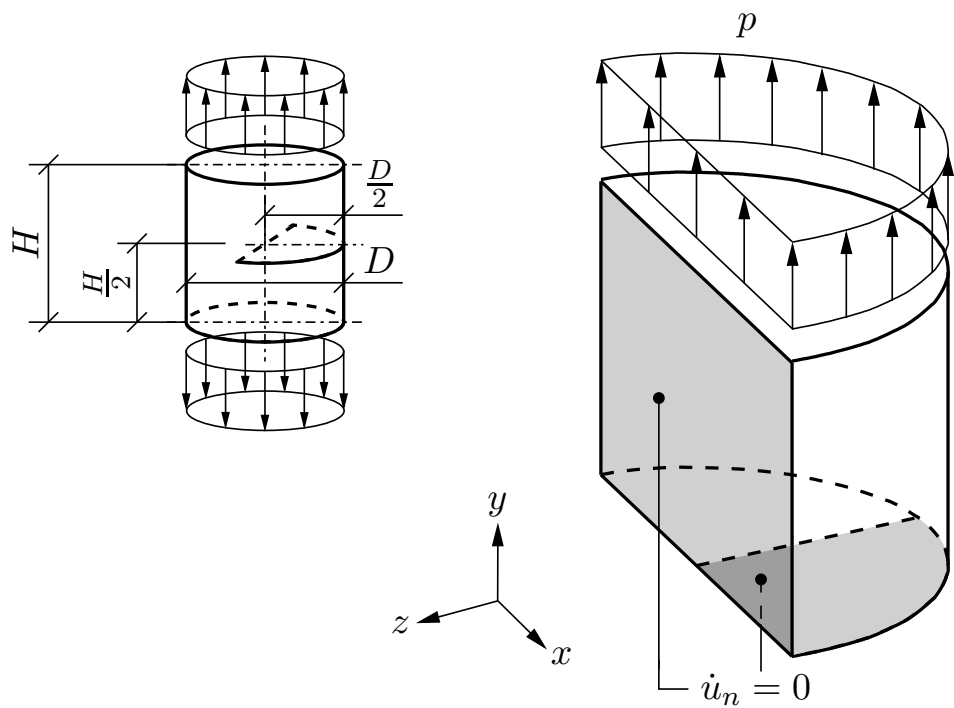

Figure 21: Example cylinder $(H=D)$ with half-circular notch under uniform tensile loading $p$, and quarter cylinder used for upper bound calculations.

load multiplier of $\beta=0.7896$. Applying the algorithm proposed in Section 6, continuous partitions cutting through the tetrahedral elements were obtained, plotted in Figure 23a using the level-set method. Figure 23b shows the resulting smooth surface, which was used to partition the original model. With this single iteration and introducing 49 discontinuities, the collapse load multiplier could already be significantly reduced $(\beta=0.7122)$. Compared to a simulation with a fine mesh (36020 elements and shown in Figures 24a and 24b) it can be concluded that the failure modes agree very well with an even slightly lower collapse load multiplier than for the fine mesh (see Table 8).

The total CPU time of the continuous approach was $11.5 \mathrm{~min}$, whereas the discontinuous approach only took 140.3 s. $26.5 \mathrm{~s}$ for the initial upper bound calculation, $79.8 \mathrm{~s}$ for the introduction of velocity discontinuities, and $34.0 \mathrm{~s}$ for the discontinuous upper bound calculation. The computational performance has been summarised in Table 8 .

\section{Summary and conclusions}

In this paper, a new implementation of finite-element-based upper bound formulations including the possibility to consider orthotropic strength behaviour is proposed, where velocity discontinuities are introduced iteratively in a sensibly way based on an adaptive introduction and adjustment algorithm.

With respect to the objectives specified in Section 1, the main findings of this paper can be summarised as follows:

1. Finite-element-based upper bound formulations have been implemented taking orthotropic strength behaviour into account and allowing velocity-jumps across sensibly-arranged discontinuities. In the discretised dual upper bound formulation, both the used stress-based yield functions assigned to solid elements and the related traction-based yield functions, describing plastic failure across velocity discontinuities, could be formulated in the form of second-order cone constraints. Thus, the resulting optimisation problems could be solved efficiently by SOCP. The obtained velocity discontinuity layouts were able to capture localised failure modes very efficiently and the obtained upper bounds agree very well with reference solutions or values obtained without discontinuities but a very fine discretisation. In this way, plastic dissipation within solid elements could be reduced significantly, making any kind of adaptive mesh refinement in failure regions obsolete.

2. The required projection of an orthotropic stress-based yield function into a locally-equivalent traction-based yield function, with respect to the orientation of an arbitrary plane, could be shown successfully and is described in detail. This algorithm has been verified numerically, showing that the failure surface in the traction basis represents a perfect envelope to the critical traction point cloud determined from critical stress states on the 


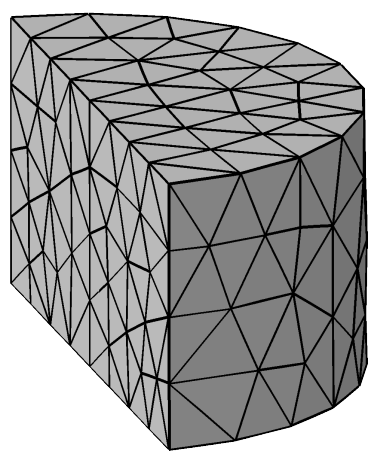

(a1) $U E=796, U D E=0$

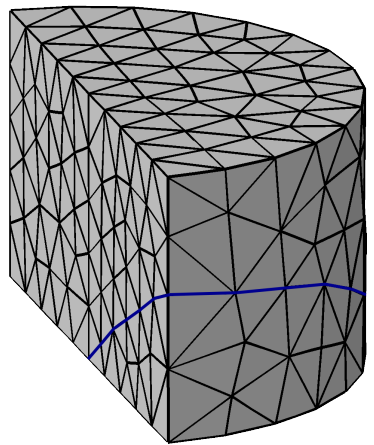

(a2) $U E=1386, U D E=49$

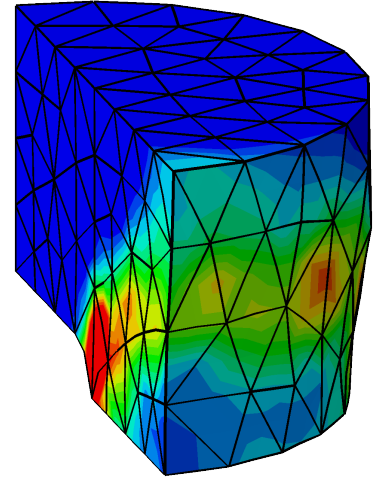

(b1) $\beta=0.7896$

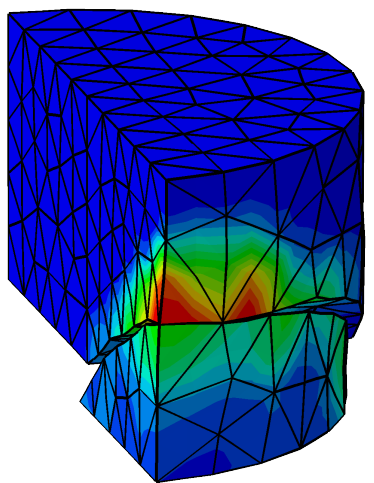

(b2) $\beta=0.7122$

Figure 22: Example cylinder with half-circular notch under uniform tensile loading, considering cohesive-frictional isotropic strength behaviour. (a1) Discretised structure used in the initial calculation; (a2) discretised structure with velocity discontinuities (blue); (b1) and (b2) related failure modes with $\dot{\lambda}_{\dot{\varepsilon}}^{\text {mat }}$ plotted in the range $\left[1 \times 10^{-6}: 1.2 \times 10^{-3}\right]$.

corresponding plane. Thus, under the assumption that plastic strain localisation with respect to a plane can occur, consistent strength behaviour throughout the whole discretised body can be guaranteed.

3. An adaptive algorithm has been implemented to iteratively introduce and arrange velocity discontinuities. At each iteration, based on the plastic multiplier rate in each strain-rate evaluation node, two critical orientations of plastic strain-rate localisation are determined. Starting from the node with the highest plastic multiplier rate, a sequence of line segments for the plane strain case and plane surfaces for the general 3D case is then generated, defining the velocity discontinuities to introduce. The so-obtained discontinuity layouts played a dominant role in the final failure mode at all investigated examples, and reliable upper bounds were obtained using relatively coarse meshes and only a limited number of velocity discontinuities.

The present approach provides an alternative method to the commonly-used numerical upper bound approaches with adaptive mesh refinement. Especially for problems where plastic failure through the body occur very localised, efficient and good upper bound solutions can be expected with a minimum number of degrees of freedom. This also applies to materials exhibiting orthotropic strength behaviour, which can be of great value for practical applications, since plastic failure in laminated structures and orthotropic materials often takes place very localised. An example would be wood at the microscale $[61,62]$ as well as at the product scale $[63,64]$.

Note that the main goal of this paper has been to propose this new concept as a further development of existing 


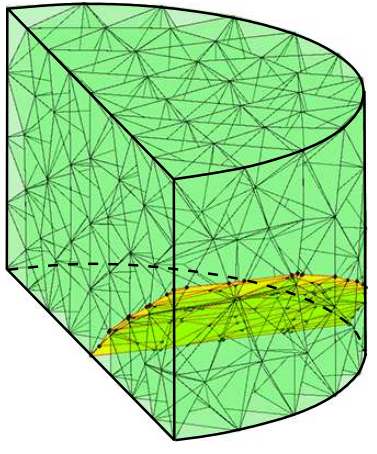

(a)

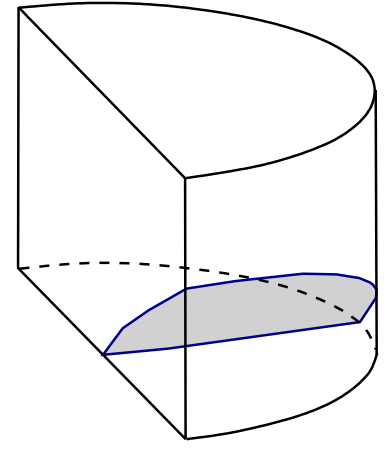

(b)

Figure 23: Example cylinder with half-circular notch under uniform tensile loading. (a) Continuous partitions cutting through tetrahedral elements defined by the level-set method introduced in Figure 6; (b) resulting smooth surface determined from the piecewise plane partition surfaces using the Face from mesh feature in ABAQUS/CAE.

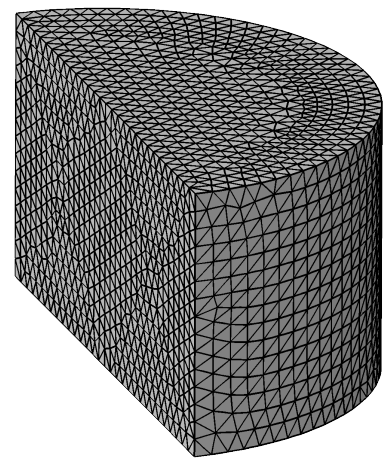

(a)

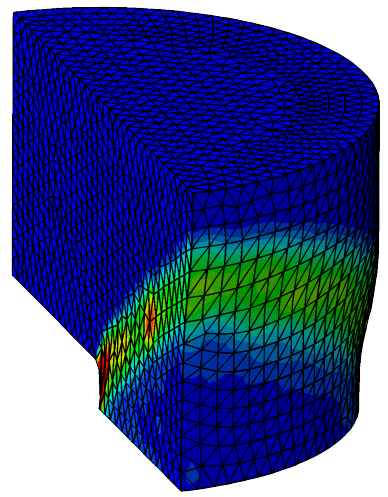

(b)

Figure 24: Example cylinder with half-circular notch under uniform tensile loading. (a) Fine mesh with 36020 tetrahedral elements and without velocity discontinuities, and (b) the corresponding continuous upper bound failure mode with the collapse load multiplier $\beta=0.7241$.

numerical upper bound formulations. The capabilities of this new approach have been assessed by means of several examples, showing high efficiency with respect to computational cost and good solution qualities. However, it is also necessary to mention that several difficulties needs to be overcome in future work. Firstly, for general 3D problems, velocity discontinuities are introduced in the discretised structures as partitions cutting through tetrahedron elements determined by means of the level-set method introduced in Section 6. When a set of partitions have been determined in the first iteration, the adjustment of existing partitions and the introduction of additional partitions in further iterations would require more advanced and comprehensive algorithms, such as the multiphase level set method, which have not yet implemented in the present approach. Secondly, for the algorithm introduced in Section 6, critical orientations can only be determined from the strain-rate states satisfying the condition in Eq. (45), derived from the assumption of strain localisation formulated in Eq. (14. This condition is fulfilled for shear-based yield functions, such as von Mises or Drucker-Prager, but seems to be too strict for other yield functions, such as the Tsai-Wu orthotropic yield function. Thus, a further development of the present algorithms is still necessary to allow a feasible and stable application for any engineering problem. 


\section{Appendix A. Second-order cone programming}

The definition of the cone set $\mathscr{C}$ is that,

$$
\forall \mathbf{x} \in \mathscr{C} \text { and } \lambda \geq 0 \Longleftrightarrow \lambda \mathbf{x} \in \mathscr{C}
$$

and then its dual cone $\mathscr{C}^{*}$ can be defined as,

$$
\mathbf{x}^{\top} \mathbf{y} \geq 0, \forall \mathbf{x} \in \mathscr{C} \Longleftrightarrow \mathbf{y} \in \mathscr{C}^{*}
$$

where $\mathbf{x} \in \mathfrak{R}^{n}$ and $\mathbf{y} \in \mathfrak{R}^{n}$. If $\mathscr{C}=\mathscr{C}^{*}$ holds, the cone is considered as self-dual.

The most common second-order cones is the quadratic cone $\mathscr{C}$,

$$
\mathscr{C}=\left\{\mathbf{x}: x_{1} \geq\left\|\mathbf{x}_{2: d}\right\|, x_{1} \geq 0\right\}
$$

and the rotated quadratic cone,

$$
\mathscr{C}=\left\{\mathbf{x}: 2 x_{1} x_{2} \geq\left\|\mathbf{x}_{3: d}\right\|, x_{1} \geq 0, x_{2} \geq 0\right\}
$$

where $\mathbf{x}_{m: n}=\left[x_{m} \ldots x_{n}\right]^{\top} \in \mathfrak{R}^{n-m}$, and both the two second-order cones are self-dual.

Then the optimization problem of the second-order cone programming (SOCP) can be expressed as

$$
\begin{array}{ll}
\min & \mathbf{c}^{\top} \mathbf{x} \\
\text { s.t. } & \mathbf{A x}=\mathbf{b} \\
& \mathbf{x} \in \mathscr{C}
\end{array}
$$

where $\mathbf{b} \in \Re^{m}, \mathbf{c} \in \Re^{n}, \mathbf{A} \in \Re^{m \times n}$ and $\mathscr{C}$ is the second-order cone.

The dual optimization problem is

$$
\begin{array}{ll}
\max & \mathbf{b}^{\top} \mathbf{z} \\
\text { s.t. } & \mathbf{A}^{\top} \mathbf{z}+\mathbf{y}=\mathbf{c} \\
& \mathbf{y} \in \mathscr{C}^{*} .
\end{array}
$$

where $\mathbf{z} \in \Re^{m}$ and $\mathscr{C}^{*}$ is the dual cone of $\mathscr{C}$.

SOCP can be regarded as a generalization of classical linear programming where the linear constraints are supplemented by specialised nonlinear constraints in the form of the cone constraints. The resulting optimization problem can be solved efficiently with primal-dual algorithms based on the interior-point method.

\section{Acknowledgments}

Financial support for this work in the framework of the PhDSchool DokInHolz funded by the Austrian Federal Ministry of Science, Research and Economy and the Austrian Association of Wood Industries is gratefully acknowledged. We also gratefully acknowledge the financial support of this work by the Austrian Science Fund (FWF) through the Erwin Schrödinger Fellowship J3748-N30.

\section{References}

[1] W. Chen, Limit analyiss and Soil plasticity, Elsevier, Amsterdam, 2013

[2] S. Sloan, Geotechnical stability analysis, Géotechnique 63 (7) (2013) 531-571.

[3] G. Milani, P. Lourenço, A. Tralli, Homogenised limit analysis of masonry walls, Part I: Failure surfaces, Computers \& Structures 84 (3) (2006) 166-180. doi:http://dx.doi.org/10.1016/j.compstruc.2005.09.005.

[4] G. Milani, E. Milani, A. Tralli, Approximate limit analysis of full scale FRP-reinforced masonry buildings through a 3D homogenized FE package, Composite Structures 92 (4) (2010) 918-935. doi:https://doi.org/10.1016/j.compstruct.2009.09.037.

[5] M. Nielsen, L. Hoang, Limit Analysis and Concrete Plasticity, 3rd Edition, Boca Raton: CRC Press, 2016.

[6] J. Füssl, R. Lackner, J. Eberhardsteiner, H. Mang, Failure modes and effective strength of two-phase materials determined by means of numerical limit analysis, Acta Mechanica 1-4 (195) (2008) 185-202. doi:10.1007/s00707-007-0550-9.

[7] H. Ciria, J. Peraire, J. Bonet, Mesh adaptive computation of upper and lower bounds in limit analysis, International Journal for Numerical Methods in Engineering 75 (8) (2008) 899-944. doi:10.1002/nme.2275.

[8] D. C. Drucker, H. J. Greenberg, W. Prager, The safety factor of an elastic-plastic body in plane strain, Journal of Applied Mechanics 18 (1951) 371-378. 
[9] D. C. Drucker, W. Prager, H. J. Greenberg, Extended limit design theorems for continuous media, Quarterly of Applied Mathematics 9 (1952) 381-389.

[10] E. Anderheggen, H. Knöpfel, Finite element limit analysis using linear programming, International Journal of Solids and Structures 8 (12) (1972) 1413-1431. doi:http://dx.doi.org/10.1016/0020-7683(72)90088-1.

[11] T. Belytschko, P. G. Hodge, Plane stress limit analysis by finite elements, Journal of the Engineering Mechanics Division 96 (6) (1970) 931-944.

[12] J. Lysmer, Limit analysis of plane problems in soil mechanics, Journal of the Soil Mechanics and Foundations Division 96 (4) (1970) 13111334.

[13] G. Maier, A. Zavelani-Rossi, D. Benedetti, A finite element approach to optimal design of plastic structures in plane stress, International Journal for Numerical Methods in Engineering 4 (4) (1972) 455-473. doi:10.1002/nme.1620040402.

[14] S. Sloan, Lower bound limit analysis using finite elements and linear programming, International Journal for Numerical and Analytical Methods in Geomechanics 12 (1) (1988) 61-77.

[15] S. Sloan, Upper bound limit analysis using finite elements and linear programming, International Journal for Numerical and Analytical Methods in Geomechanics 13 (3) (1989) 263-282.

[16] S. Sloan, P. Kleeman, Upper bound limit analysis using discontinuous velocity fields, Computer Methods in Applied Mechanics and Engineering 127 (14) (1995) 293-314. doi:10.1016/0045-7825(95)00868-1.

[17] A. V. Lyamin, S. W. Sloan, Lower bound limit analysis using non-linear programming, International Journal for Numerical Methods in Engineering 55 (5) (2002) 573-611. doi:10.1002/nme.511.

[18] A. V. Lyamin, S. W. Sloan, Upper bound limit analysis using linear finite elements and non-linear programming, International Journal for Numerical and Analytical Methods in Geomechanics2 26 (2) (2002) 181-216. doi:10.1002/nag.198.

[19] K. Krabbenhøft, L. Damkilde, A general non-linear optimization algorithm for lower bound limit analysis, International Journal for Numerical Methods in Biomedical Engineering 56 (2003) 165-187.

[20] A. Makrodimopoulos, C. M. Martin, Lower bound limit analysis of cohesive-frictional materials using second-order cone programming, International Journal for Numerical Methods in Engineering 66 (4) (2006) 604-634. doi:10.1002/nme.1567.

[21] A. Makrodimopoulos, C. M. Martin, Upper bound limit analysis using simplex strain elements and second-order cone programming, International Journal for Numerical and Analytical Methods in Geomechanics 31 (6) (2007) 835-865. doi:10.1002/nag.567.

[22] H. Ciria, J. Peraire, Computation of upper and lower bounds in limit analysis using second-order cone programming and mesh adaptivity, in: 9th ASCE Specialty Conference on Probabilistic Mechanics and Structural Reliability, 2004.

[23] K. Krabbenhøft, A. Lyamin, S. Sloan, Formulation and solution of some plasticity problems as conic programs, International Journal of Solids and Structures 44 (5) (2007) 1533-1549. doi:https://doi.org/10.1016/j.ijsolstr.2006.06.036

[24] A. Bottero, R. Negre, J. Pastor, S. Turgeman, Finite element method and limit analysis theory for soil mechanics problems, Computer Methods in Applied Mechanics and Engineering 22 (1) (1980) 131-149. doi:http://dx.doi.org/10.1016/0045-7825(80)90055-9.

[25] C. Martin, A. Makrodimopoulos, Finite-element limit analysis of Mohr-Coulomb materials in 3D using semidefinite programming, Journal of Engineering Mechanics 134 (4) (2008) 339-347. arXiv:http://dx.doi.org/10.1061/(ASCE)0733-9399(2008)134:4(339), doi:10.1061/(ASCE)0733-9399(2008)134:4(339).

[26] C. V. Le, M. Gilbert, H. Askes, Limit analysis of plates using the EFG method and second-order cone programming, International Journal for Numerical Methods in Engineering 78 (13) (2009) 1532-1552. arXiv:https://onlinelibrary.wiley.com/doi/pdf/10.1002/nme.2535, doi:10.1002/nme.2535.

[27] C. V. Le, M. Gilbert, H. Askes, Limit analysis of plates and slabs using a meshless equilibrium formulation, International Journal for Numerical Methods in Engineering 83 (2010) 1739-1758.

[28] F. Liu, J. Zhao, Upper bound limit analysis using radial point interpolation meshless method and nonlinear programming, International Journal of Mechanical Sciences 70 (2013) 26-38. doi:http://dx.doi.org/10.1016/j.ijmecsci.2013.01.017.

[29] S. Yu, X. Zhang, S. Sloan, A 3D upper bound limit analysis using radial point interpolation meshless method and second-order cone programming, International Journal for Numerical Methods in Engineering 108 (13) (2016) 1686-1704.

[30] C. V. Le, H. Nguyen-Xuan, H. Askes, S. P. A. Bordas, T. Rabczuk, H. Nguyen-Vinh, A cell-based smoothed finite element method for kinematic limit analysis, International Journal for Numerical Methods in Engineering 83 (12) (2010) $1651-1674$. arXiv:https://onlinelibrary.wiley.com/doi/pdf/10.1002/nme.2897, doi:10.1002/nme.2897.

[31] H. Nguyen-Xuan, G. Liu, An edge-based finite element method (ES-FEM) with adaptive scaled-bubble functions for plane strain limit analysis, Computer Methods in Applied Mechanics and Engineering 285 (Supplement C) (2015) 877-905. doi:https://doi.org/10.1016/j.cma.2014.12.014.

[32] K. Krabbenhøft, A. V. Lyamin, M. Hjiaj, S. W. Sloan, A new discontinuous upper bound limit analysis formulation, International Journal for Numerical Methods in Engineering 63 (7) (2005) 1069-1088. doi:10.1002/nme.1314.

[33] A. Makrodimopoulos, C. M. Martin, Upper bound limit analysis using discontinuous quadratic displacement fields, Communications in Numerical Methods in Engineering 24 (11) (2008) 911-927. doi:10.1002/cnm.998.

[34] J. Chen, J.-H. Yin, C. Lee, Upper bound limit analysis of slope stability using rigid finite elements and nonlinear programming, Canadian Geotechnical Journal 40 (2003) 742-752.

[35] G. Milani, P. Lourenço, A. Tralli, Homogenised limit analysis of masonry walls, Part II: Structural examples, Computers \& Structures 84 (3) (2006) 181-195. doi:http://dx.doi.org/10.1016/j.compstruc.2005.09.004.

[36] G. Milani, P. Lourenço, A discontinuous quasi-upper bound limit analysis approach with sequential linear programming mesh adaptation, International Journal of Mechanical Sciences 51 (2009) 89-104.

[37] C. Smith, M. Gilbert, Application of discontinuity layout optimization to plane plasticity problems, Proceedings of the Royal Society A 463 (2007) 2461-2484.

[38] S. Hawksbee, C. Smith, M. Gilbert, Application of discontinuity layout optimization to three-dimensional plasticity problems, Proceedings of the Royal Society of London A: Mathematical, Physical and Engineering Sciences 469 (2155). arXiv:http://rspa.royalsocietypublishing.org/content/469/2155/20130009.full.pdf, doi:10.1098/rspa.2013.0009. 
[39] L. Borges, N. Zouain, C. Costa, R. Feijóo, An adaptive approach to limit analysis, International Journal of Solids and Structures 38 (10) (2001) 1707-1720. doi:https://doi.org/10.1016/S0020-7683(00)00131-1.

[40] F. Tin-Loi, N. Ngo, Performance of the p-version finite element method for limit analysis, International Journal of Mechanical Sciences 45 (6) (2003) 1149 - 1166. doi:https://doi.org/10.1016/j.ijmecsci.2003.08.004.

[41] J. J. Muñoz, J. Bonet, A. Huerta, J. Peraire, Upper and lower bounds in limit analysis: Adaptive meshing strategies and discontinuous loading, International Journal for Numerical Methods in Engineering 77 (4) (2009) 471-501. arXiv:https://onlinelibrary.wiley.com/doi/pdf/10.1002/nme.2421, doi:10.1002/nme.2421.

[42] C. Martin, The use of adaptive finite-element limit analysis to reveal slip-line fields, Géotechnique Letters 1 (2) (2011) $23-29$. doi:10.1680/geolett.11.00018

[43] H. Nguyen-Xuan, S. Nguyen-Hoang, T. Rabczuk, K. Hackl, A polytree-based adaptive approach to limit analysis of cracked structures, Computer Methods in Applied Mechanics and Engineering 313 (Supplement C) (2017) 1006-1039. doi:https://doi.org/10.1016/j.cma.2016.09.016.

[44] M. Li, J. Füssl, M. Lukacevic, J. Eberhardsteiner, C. M. Martin, A numerical upper bound formulation with sensible-arranged velocity discontinuities and orthotropic material strength behaviour, Journal of Theoretical and Applied Mechanics 56 (2) (2018) $417-433$.

[45] A. Capsoni, L. Corradi, P. Vena, Limit analysis of orthotropic structures based on hill's yield condition, International Journal of Solids and Structures 38 (22-23) (2001) 3945-3963. doi:10.1016/S0020-7683(00)00241-9.

[46] A. Capsoni, L. Corradi, P. Vena, Limit analysis of anisotropic structures based on the kinematic theorem, International Journal of Plasticity 17 (11) (2001) 1531-1549. doi:10.1016/S0749-6419(01)00023-7.

[47] H. Li, Limit analysis of composite materials with anisotropic microstructures: A homogenization approach, Mechanics of Materials 43 (10) (2011) 574-585. doi:10.1016/j.mechmat.2011.06.007.

[48] J. Füssl, M. Li, M. Lukacevic, J. Eberhardsteiner, C. M. Martin, Comparison of unit cell-based computational methods for predicting the strength of wood, Engineering Structures 141 (2017) 427-443.

[49] M. Li, J. Füssl, M. Lukacevic, J. Eberhardsteiner, C. M. Martin, Strength predictions of clear wood at multiple scales using numerical limit analysis approaches, Computers \& Structures 196 (2018) 200-216.

[50] M. Li, J. Füssl, M. Lukacevic, C. M. Martin, J. Eberhardsteiner, Bending strength predictions of cross-laminated timber plates subjected to concentrated loading using 3D finite-element-based limit analysis approaches, Composite Structures.

[51] J.-Y. Wu, M. Cervera, On the equivalence between traction- and stress-based approaches for the modeling of localized failure in solids, Journal of the Mechanics and Physics of Solids 82 (2014) 137-163.

[52] K. Krabbenhøft, A. Lyamin, S. Sloan, Three-dimensional MohrCoulomb limit analysis using semidefinite programming, Communications in Numerical Methods in Engineering 24 (2008) 1107-1119.

[53] A. Makrodimopoulos, Remarks on some properties of conic yield restrictions in limit analysis, International Journal for Numerical Methods in Biomedical Engineering 26 (11) (2010) 1449-1461. doi:10.1002/cnm.1224.

[54] S. Osher, J. A. Sethian, Fronts propagating with curvature-dependent speed: Algorithms based on Hamilton-Jacobi formulations, Journal of Computational Physics 79 (1) (1988) 12-49.

URL http: //www.sciencedirect.com/science/article/pii/0021999188900022

[55] N. Moës, A. Gravouil, T. Belytschko, Non-planar 3D crack growth by the extended finite element and level sets-Part I: Mechanical model, International Journal for Numerical Methods in Engineering 53 (11) (2002) 2549-2568.

[56] MOSEK ApS, The MOSEK optimization tools version 8.0, User's manual and reference, available from http://www.mosek.com [June 2014] (2017).

[57] E. Andersen, C. Roos, T. Terlaky, Notes on duality in second order and p-order cone optimization, Optimization 51 (2002) $627-643$.

[58] E. Christiansen, K. D. Andersen, Computation of collapse states with von mises type yield condition, International Journal for Numerical Methods in Engineering 46 (8) (1999) 1185-1202.

[59] E. Christiansen, O. S. Pedersen, Automatic mesh refinement in limit analysis, International Journal for Numerical Methods in Engineering 50 (6) (2001) 1331-1346.

[60] M. Dorn, Investigations on the serviceability limit state of dowel-type timber connections, Ph.D. thesis, Vienna University of Technology (2012).

[61] M. Lukacevic, J. Füssl, R. Lampert, Failure mechanisms of clear wood identified at wood cell level by an approach based on the extended finite element method, Engineering Fracture Mechanics 144 (2014) 158-175. doi:10.1016/j.engfracmech.2015.06.066.

[62] M. Lukacevic, J. Füssl, Application of a multisurface discrete crack model for clear wood taking into account the inherent microstructural characteristics of wood cells, Holzforschung 70 (9) (2016) 845-853.

[63] G. Hochreiner, J. Füssl, J. Eberhardsteiner, Cross-laminated timber plates subjected to concentrated loading - Experimental identification of failure mechanisms, Strain 50 (1) (2013) 68-81. doi:10.1111/str.12068.

[64] G. Hochreiner, J. Füssl, E. Serrano, J. Eberhardsteiner, Influence of wooden board strength class on the performance of cross-laminated timber plates investigated by means of full-field deformation measurements, Strain 50 (2) (2014) 161-173. doi:10.1111/str.12077. 